\title{
Một phương pháp mới nâng cao độ tương phản ảnh màu theo hướng tiếp cận trực tiếp
}

\section{A New Method to Enhancement The Contrast of Color Images based on Direct Method}

\author{
Nguyễn Văn Quyền, Trần Thái Sơn, Nguyễn Tân Ân, Ngô Hoàng Huy, Đặng Duy An
}

\begin{abstract}
Image contrast enhancement techniques have two mainly methods: indirect method and direct method. While indirect methods only modify the histogram without defining any specific contrast measure, the direct methods establish a criterion of contrast measurement and enhance the image by improving the contrast measure. Among many direct methods, only the studies by Cheng and Xu modified the contrast at each point of grayscale image using a contrast measure [6, 7].

In this paper we propose a new method for enhancing the contrast of color images based on the direct method. The experimental results demonstrate that the combination of our proposed method with Fuzzy C_Mean (FCM) clustering algorithms performs well on different color images.
\end{abstract}

Keywords: Direct contrast enhancement, homogeneity measure, contrast measure, FCM, Sfunction, histogram, the dynamic range of gray, $H S V$, entropy, fuzzy entropy, the image details.

\section{GIỚI THIỆU}

Nâng cao độ tương phản ảnh là một vấn đề quan trọng trong xử lý và phân tích hình ảnh, là một bước cơ bản trong phân đoạn ảnh. Các kỹ thuật thông dụng nâng cao độ tương phản ảnh được phân loại theo hai tiếp cận chính: (1) Các phương pháp gián tiếp $[1,2,5$, $12,13,17]$; và (2) các phương pháp trực tiếp [6, 7].

Có rất nhiều kỹ thuật đã được đề xuất được tìm thấy trong tài liệu tham khảo, hầu hết trong số đó là phương pháp gián tiếp, chúng biến đổi histogram mà không sử dụng bất kỳ một độ đo tương phản nào.
Các kỹ thuật biến đổi histogram được khai thác ở nhiều khía cạnh như thuật toán khung biến đổi histogram và thích nghi nội dung [2], biến đổi logarit histogram [1], kỹ thuật cân bằng động histogram [5], chuẩn hóa nhiều histogram [17], biến đổi Cosine rời rạc [13], xây dựng toán tử tăng cường mở rộng của toán tử INT của Zadeh để mờ hóa thông tin trong miền không gian [12] v.v...

Mặc dù vậy có rất ít các nghiên cứu theo phương pháp trực tiếp trong đó biến đổi độ tương phản ảnh dựa trên một độ đo tương phản xác định tại mỗi điểm ảnh, chẳng hạn các nghiên cứu $[9,3,8,6,7]$. Trong $[9,3]$, các kết quả đã chứng tỏ rằng phương pháp trực tiếp có thể tạo ra các kỹ thuật nâng cao độ tương phản hiệu quả.

Như nhận xét ở trên, trong một thời gian dài cho đến nay hầu như chỉ có các nghiên cứu của Cheng và Xu $[6,7]$ là đề xuất một phương pháp biến đổi độ tương phản tại mỗi điểm ảnh dựa trên định nghĩa một độ đo tương phản giữa độ sáng điểm ảnh và lân cận xung quanh nó. Độ đo tương phản của $[6,7]$ được xây dựng dựa trên các đặc trưng địa phương như gradient, entropy, độ lệch chuẩn trung bình và moment bậc 4 tại từng điểm ảnh.

Ngay từ đầu, phương pháp nâng cao độ tương phản này chỉ được thực hiện trong ảnh đa cấp xám. Mỏ rộng những phương pháp này để nâng cao độ tương phản của ảnh màu không phải là một nhiệm vụ dễ dàng do gặp phải một số yếu tố, chẳng hạn như sự lựa chọn một mô hình màu thích hợp để biểu diễn và xử lý ảnh, ảnh hưởng hệ thống thị giác của con người. 
Việc lựa chọn một mô hình màu là rất quan trọng để cải thiện độ tương phản của ảnh màu. Biểu diễn màu RGB được sử dụng cho việc hiển thị màu sắc, trong khi biểu diễn màu HSV là cho hệ thống thị giác của con người. Trong mô hình màu HSV, kênh $\mathrm{H}$ biểu diễn màu sắc, kênh $\mathrm{S}$ chỉ độ bão hòa, và kênh $\mathrm{V}$ là cường độ của màu. Bằng cách bảo toàn kênh $\mathrm{H}$, chỉ thay đổi kênh $\mathrm{V}$ hoặc thay đổi cả kênh $\mathrm{S}$ và kênh $\mathrm{V}$, chúng ta có thể nâng cao chất lượng ảnh màu mà không làm suy giảm chất lượng ảnh gốc [12].

Trong khi các thuật toán trong $[6,7]$ là cơ sở của phép nâng cao độ tương phản các ảnh đa cấp xám, chúng không đảm bảo hiệu quả khi áp dụng trực tiếp cho ảnh màu do một số nguyên nhân sau:

(i) Ảnh nâng cao độ tương phản không thay đổi mức độ sáng của màu so với ảnh gốc.

Khi áp dụng thuật toán [7] trên kênh $\mathrm{V}$ của ảnh màu trong biểu diễn màu HSV, chúng tôi nhận thấy với nhiều ảnh màu, đặc biệt là các ảnh tối, các điểm ảnh như vậy có thể chiếm rất nhiều. Do đó ảnh được nâng cao sẽ không thay đổi mức sáng ở những vùng này và không khác biệt với ảnh gốc nếu chỉ dựa trên cảm nhận bằng mắt.

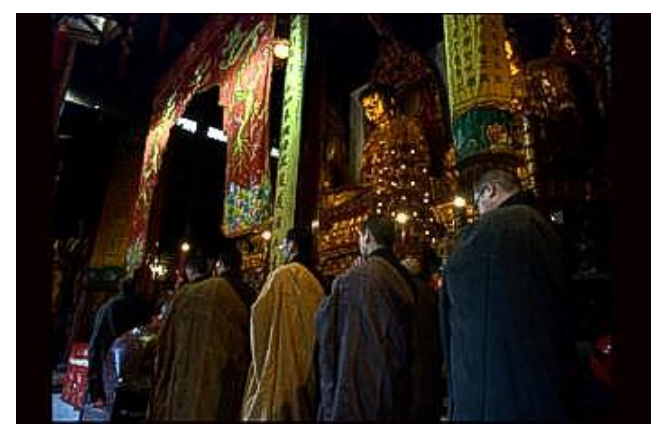

Hình 1. Ảnh kết quả khi sủ dụng [7] cho ảnh \#1 (Xem hinh 5)

(ii) Chi tiết của ảnh gốc bị suy giảm.

Trong [6], các tác giả đề xuất một thuật toán sử dụng hàm $\mathrm{S}$-function có tham số để biến đổi ảnh đa cấp xám I đầu vào sau đó nâng cao độ tương phản của ảnh biến đổi theo phương pháp trực tiếp.

$I=\{I(i, j)\} \mapsto S I\left(a, b_{\text {opp }}, c\right)=\left\{S-f u n c\left(I(i, j) ; a, b_{\text {opt }}, c\right)\right\}$, trong đó $\mathbf{a}, \mathrm{b}_{\mathrm{opt}}$ và $\mathbf{c}$ là các tham số được ước lượng tự động khi khảo sát các đỉnh histogram và dựa trên nguyên lý cực đại fuzzy entropy:

$$
b_{o p t}=\underset{b \in[a+1, c-1]}{\arg \max }\{H(I ; a, b, c)\},
$$

và $H$ là độ đo fuzzy entropy thông dụng (xem công thức (33), mục IV.3).

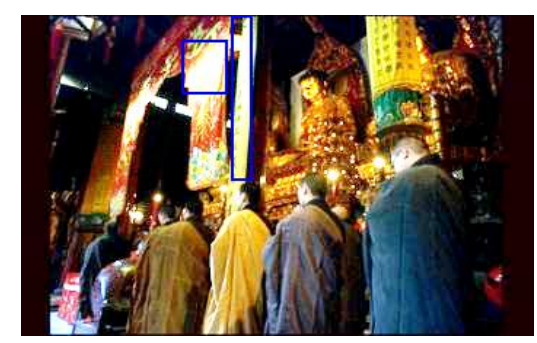

Hình 2. Anh biến đổi sủ dụng hàm S-function bị mất chi tiết [6].

Hình 2 chứng tỏ khi áp dụng phép biến đổi dạng $\mathrm{S}$ function ở trên cho từng kênh $R, G$ và $B$ của ảnh \#1 chúng ta sẽ nhận được một ảnh bị mất chi tiết như đã được thể hiện ở vùng đánh dấu chữ nhật. Điều này cũng xảy ra khi áp dụng biến đổi này cho kênh $\mathrm{V}$ trong biểu diễn màu HSV của ảnh \#1.

Phần còn lại của bài báo được tổ chức như sau: Phần II, trình bày một số nghiên cứu liên quan của thuật toán nâng cao độ tương phản theo hướng trực tiếp của Cheng và cộng sự; Phần III là đề xuất thuật toán sử dụng phân cụm mờ để ước lượng nhiều khoảng động mức xám, xây dựng hàm biến đổi kênh ảnh trước khi tính độ tương phản điểm ảnh và thuật toán nâng cao độ tương phản ảnh màu trong biểu diễn màu HSV; Các kết quả thực nghiệm trình bày trong phần IV; Kết luận được đưa ra ở phần V.

\section{NGHIÊN CÚU LIÊN QUAN}

Bảng 1 liệt kê một số kí hiệu được sử dụng trong bài báo này.

Bảng 1. Các ký hiệu và các định nghĩa của nó

\begin{tabular}{|c|l|}
\hline Ký hiệu & \multicolumn{1}{|c|}{ Định nghĩa } \\
\hline $\mathrm{I}$ & Ảnh RGB nói chung \\
\hline $\mathrm{M}, \mathrm{N}$ & $\begin{array}{l}\text { M x N là kích thước theo pixel của ảnh } \\
\text { đầu vào. }\end{array}$ \\
\hline $\mathrm{I}_{\mathrm{R}}, \mathrm{I}_{\mathrm{G}}, \mathrm{I}_{\mathrm{B}}$ & $\begin{array}{l}\text { Kênh ảnh R,G và } \mathrm{B} \text { của ảnh màu trong } \\
\text { biểu diễn màu RGB. }\end{array}$ \\
\hline
\end{tabular}




\begin{tabular}{|c|c|}
\hline $\mathrm{I}_{\mathrm{S}}, \mathrm{I}_{\mathrm{H}}, \mathrm{I}_{\mathrm{V}}$ & $\begin{array}{l}\text { Kênh ảnh } \mathrm{H}, \mathrm{S} \text { và } \mathrm{V} \text { của ảnh màu trong } \\
\text { biểu diê̂n màu HSV }\end{array}$ \\
\hline $\begin{array}{l}\mathrm{L}_{\mathrm{k}, \min } \\
\mathrm{L}_{\mathrm{k}, \max }\end{array}$ & $\begin{array}{l}\text { Miền giá trị mức xám của kênh ảnh thứ } \\
\mathrm{k} \text { của ảnh đầu vào, thông thường } \mathrm{L}_{\mathrm{k}, \text { min }} \\
=0, \mathrm{~L}_{\mathrm{k}, \max }=255 \text {. }\end{array}$ \\
\hline d & $\begin{array}{l}\mathrm{dx} \text { d là kích thước cửa sổ lân cận của } \\
\text { điểm ảnh. }\end{array}$ \\
\hline $\mathrm{E}_{\mathrm{ij}}$ & $\begin{array}{l}\text { Các giá trị gradient lấy tại điểm ảnh (i, } \\
\text { j) được chuẩn hóa về miền }[0,1] \text { theo } \\
\text { một toán tử tìm kiếm biên chẳng hạn } \\
\text { toán tử Sobel. }\end{array}$ \\
\hline $\mathrm{H}_{\mathrm{ij}}$ & $\begin{array}{l}\text { Giá trị entropy địa phương lấy tại điểm } \\
\text { ảnh }(i, j) \text { được chuẩn hóa về miền }[0,1] \text {. }\end{array}$ \\
\hline $\mathrm{V}_{\mathrm{ij}}$ & $\begin{array}{l}\text { Độ lệch chuẩn trung bình mức xám lấy } \\
\text { tại điểm ảnh }(i, j) \text { được chuẩn hóa về } \\
\text { miền }[0,1]\end{array}$ \\
\hline $\mathrm{R}_{4, \mathrm{ij}}$ & $\begin{array}{l}\text { Giá trị moment bậc } 4 \text { lấy tại điểm ảnh } \\
(\mathrm{i}, \mathrm{j}) \text { được chuẩn hóa về miền }[0,1]\end{array}$ \\
\hline $\mathrm{HO}_{\mathrm{ij}}$ & $\begin{array}{l}\text { Giá trị kết nhập dạng } \mathrm{f}\left(\mathrm{E}_{\mathrm{ij}}, \mathrm{H}_{\mathrm{ij}}, \mathrm{V}_{\mathrm{ij}} \text {, }\right. \\
\left.\mathrm{R}_{4, \mathrm{ij}}\right)[7]\end{array}$ \\
\hline$\beta_{\mathrm{ij}}$ & Giá trị thuần nhất tại điểm ảnh $(\mathrm{i}, \mathrm{j})$ \\
\hline$\delta_{\mathrm{ij}}$ & $\begin{array}{l}\text { Giá trị trung bình không thuần nhất tại } \\
\text { điểm ảnh }(i, j)\end{array}$ \\
\hline$\xi_{\mathrm{ij}}$ & Số mũ khuếch đại tại $(\mathrm{i}, \mathrm{j})$ \\
\hline $\mathrm{t}$ & $\begin{array}{l}\mathrm{t} \in(0,1) \text { : Tham số của phép nâng độ } \\
\text { khuếch đại }\end{array}$ \\
\hline $\mathrm{f}_{1}, \mathrm{f}_{2}$ & $\begin{array}{l}\mathrm{f}_{1}, \mathrm{f}_{2} \in(0,1) \text { : Tham số xác định dải } \\
\text { động mức xám [7] }\end{array}$ \\
\hline $\mathrm{K}$ & Số kênh ảnh cần xử lý của ảnh đầu vào \\
\hline $\mathrm{C}$ & $\begin{array}{l}\text { Số cụm cần phân cụm của tổ hợp kênh } \\
\text { ảnh đầu vào. }\end{array}$ \\
\hline$\mu_{\mathrm{i}, \mathrm{j}, \mathrm{c}}$ & $\begin{array}{l}\text { Giá trị độ thuộc cụm thứ } \mathbf{c} \text { của điểm ảnh } \\
(i, j) \text {, dầu ra của thủ tục phân cụm FCM }\end{array}$ \\
\hline$f_{\text {cut }}$ & $\begin{array}{l}\mathrm{f}_{\text {cut }} \in(0,1) \text { : Tham số xác định } \mathrm{C} \text { dải } \\
\text { động mức xám của một kênh ảnh (mục } \\
\text { III) }\end{array}$ \\
\hline
\end{tabular}

\section{II.1. Độ tương phản trực tiếp tại từng điểm ảnh}

Thông thường, độ tương phản chỉ sự chênh lệch về độ sáng giữa một đối tượng (ký hiệu là $f$ ) và vùng xung quanh của nó (ký hiệu là b). Tương tự như [6, 7], trong bài báo này chúng ta cũng sử dụng độ đo tương phản sau:

$$
C=\left|\frac{f-b}{f+b}\right|
$$

Nâng cao độ tương phản theo phương pháp trực tiếp, theo $[6,7]$ là việc thực hiện một dãy biển đổi $(f, b) \mapsto C_{f, b} \mapsto C_{\text {new }} \mapsto f_{\text {new, }, b}, 0 \leq \mathrm{C}_{\mathrm{f}, \mathrm{b}} \leq \mathrm{C}_{\text {new }} \leq 1$ và $f_{\text {new }, f, b}=\left\{\begin{array}{l}b \frac{1-C_{\text {new }}}{1+C_{\text {new }}}, f \leq b \\ b \frac{1+C_{\text {new }}}{1-C_{\text {new }}}, f>b\end{array}\right.$

\section{II.2. S-function}

Trong lý thuyết tập mờ, Zadeh đã định nghĩa một toán tử tăng cường gọi là INT (intensification), và dạng tổng quát của nó được gọi là S-function [7], được xác định như sau:

$$
S(x ; a, b, c)=\left\{\begin{array}{l}
0,0 \leq x<a \\
\frac{(x-a)^{2}}{(b-a)(c-a)}, a \leq x \leq b \\
1-\frac{(x-c)^{2}}{(c-b)(c-a)}, b \leq x \leq c \\
1, c \leq x
\end{array}\right.
$$

Trong [6] Cheng và cộng sự đã sử dụng hàm $\mathrm{S}$ function để chuyển một ảnh xám sang miền fuzzy, sau đó nghiên cứu nâng cao độ tương phản của ảnh trong miền fuzzy.

Trong [7] đề xuất xây dựng phép nâng cao độ tương phản trực tiếp của ảnh đa cấp xám dựa trên các đặc trưng địa phương của điểm ảnh. Đây là phương pháp gốc được dùng phát triển các thuật toán sẽ được đề xuất trong phần III.

\section{II.3. Ước lượng độ sáng nền và độ tương phản điểm ảnh}

Phép nâng cao độ tương phản trực tiếp của một ảnh xám được công bố trong [7] có thể được tóm tắt như sau:

Giả sử $\mathrm{g}_{\mathrm{ij}}$ là mức xám của một điểm ảnh $\mathrm{I}(\mathrm{i}, \mathrm{j})$ at của ảnh đa cấp xám I kích thước $\mathrm{M} \times \mathrm{N}$, và $\mathrm{W}_{\mathrm{ij}}$ cửa sổ lân cận tại $(i, j)$ kích thước $\mathrm{d} \times \mathrm{d}$. Thực hiện tuần tự các bước sau:

Bước 1: Tính các tham số địa phương được chuẩn hóa giá trị về đoạn $[0,1]$, gradient $E_{i j}$, entropy $H_{i j}$, trung bình độ lệch chuẩn $V_{i j}$, và moment bậc $4 R_{4, i j}$ :

\section{1: Tính cường độ biên ảnh:}

$\mathrm{e}=\left\{\mathrm{e}_{\mathrm{ij}}\right\}$ là giá trị cường độ biên ảnh xám đầu vào bằng một toán tử xác định ảnh biên như toán tử Sobel. 
1.2: Giá trị trung bình, độ lệch chuẩn

$\mu=\left\{\mu_{\mathrm{ij}}\right\}, \mathrm{v}=\left\{\mathrm{v}_{\mathrm{ij}}\right\}$, trong đó $\mu_{\mathrm{ij}}$ là giá trị trung bình mức xám, $\mathrm{v}_{\mathrm{ij}}$ là độ lệch chuẩn mức xám lấy tại lân cận điểm ảnh $g_{i j}$

$$
\mu_{\mathrm{ij}}=\frac{\sum_{(p, q) \in \mathrm{W}_{\mathrm{ij}}} g_{p q}}{d^{2}}, v_{\mathrm{ij}}=\sqrt{\frac{\sum_{(p, q) \in \mathrm{W}_{\mathrm{ij}}}\left(g_{p q}-\mu_{\mathrm{ij}}\right)^{2}}{d^{2}}}
$$

1.3: Tính giá trị entropy địa phương

$$
\begin{gathered}
h_{\mathrm{ij}}=\frac{\sum_{k=1}^{L} p_{k} \log p_{k}}{2 \log d}, \\
p_{k}=\frac{\#\left\{(p, q) \in \mathrm{W}_{\mathrm{ij}}: g_{p, q}=k\right\}}{d^{2}}
\end{gathered}
$$

1.4: Tính moment bậc 4

$$
\gamma_{4, \mathrm{ij}}=\frac{\sum_{(p, q) \in \mathrm{W}_{\mathrm{ij}}}\left(g_{p q}-\mu_{\mathrm{ij}}\right)^{4}}{d^{2}-1}
$$

1.5: Chuẩn hóa về miền giá trị là đoạn $[0,1]$

$$
\begin{aligned}
E_{\mathrm{ij}} & =\frac{e_{\mathrm{ij}}}{\max \left\{e_{\mathrm{ij}}\right\}}, V_{\mathrm{ij}}=\frac{v_{\mathrm{ij}}}{\max \left\{v_{\mathrm{ij}}\right\}} \\
H_{i j} & =\frac{h_{\mathrm{ij}}}{\max \left\{h_{\mathrm{ij}}\right\}}, R_{4, i j}=\frac{\gamma_{4, \mathrm{ij}}}{\max \left\{\gamma_{4, \mathrm{ij}}\right\}}
\end{aligned}
$$

Bước 2: Tính giá trị độ thuần nhất của điểm ảnh và giá trị mức xám không thuần nhất

2.1: Tính giá trị đo độ thuần nhất tại điểm ảnh

$$
\beta_{\mathrm{ij}}=\frac{H O_{\mathrm{ij}}}{\max H O_{\mathrm{ij}}},
$$

trong đó

$$
\begin{aligned}
H O_{i j} & =\overline{E_{\mathrm{ij}}} * \overline{V_{i j}} * \overline{H_{i j}} * \overline{R_{4 \partial}} \\
& =\left(1-E_{\mathrm{ij}}\right) *\left(1-V_{i j}\right) *\left(1-H_{i j}\right) *\left(1-R_{4, i j}\right)
\end{aligned}
$$

2.2: Tính giá trị mức xám không thuần nhất (nonhomogeneity gray value [7])

$$
\delta_{\mathrm{ij}}=\frac{\sum_{(p, q) \in \mathrm{W}_{\mathrm{ij}}} g_{p q}\left(1-\beta_{p q}\right)}{\sum_{(p, q) \in \mathrm{W}_{\mathrm{ij}}}\left(1-\beta_{p q}\right)}
$$

Bước 3: Tính giá trị độ tương phản tại từng điểm ảnh và số mũ khuếch đại

3.1: Giá trị độ tương phản

3.2: Số mũ khuếch đại

$$
\xi_{\mathrm{ij}}=\xi_{\text {min }}+\frac{\left(\xi_{\text {max }}-\xi_{\text {min }}\right) *\left(\beta_{\mathrm{ij}}-\beta_{\text {min }}\right)}{\beta_{\text {max }}-\beta_{\text {min }}},
$$

trong đó: $\xi_{\text {min }}=\frac{g_{k}-g_{1}}{g_{\text {max }}-g_{1}}, \xi_{\max }=1, \mathrm{~g}_{\mathrm{k}}, \mathrm{g}_{1}$

là các đỉnh của histogram được xác định theo [3]

3.3: Nâng độ tương phản

$$
C_{\mathrm{ij}}^{\prime}=C_{\mathrm{ij}}^{\xi_{\mathrm{ij}}^{t}},
$$

trong đó tham số $\mathrm{t} \in\{0.25,0.5\}$ [7]

3.4: Tính giá trị mức xám mới tại từng điểm ảnh

$$
g_{\mathrm{ij}}^{\prime}=\left\{\begin{array}{l}
\delta_{\mathrm{ij}} \frac{1-C_{\mathrm{ij}}^{\prime}}{1+C_{\mathrm{ij}}^{\prime}}=\delta_{\mathrm{ij}} \frac{1-C_{\mathrm{ij}}^{\xi_{\mathrm{j}}^{t}}}{1+C_{\mathrm{ij}}^{\xi_{\mathrm{j}}^{\prime}}}, g_{\mathrm{ij}} \leq \delta_{\mathrm{ij}} \\
\delta_{\mathrm{ij}} \frac{1+C_{\mathrm{ij}}^{\prime}}{1-C_{\mathrm{ij}}^{\prime}}=\delta_{\mathrm{ij}} \frac{1+C_{\mathrm{ij}}^{\xi_{\mathrm{j}}^{\prime}}}{1-C_{\mathrm{ij}}^{\xi_{\mathrm{j}}^{\prime}}}, g_{\mathrm{ij}}>\delta_{\mathrm{ij}}
\end{array}\right.
$$

Thuật toán $[6,7]$ thỏa mãn điều kiện sau: Tại từng điểm ảnh, độ thuần nhất càng cao thì mức độ nâng tương phản càng thấp.

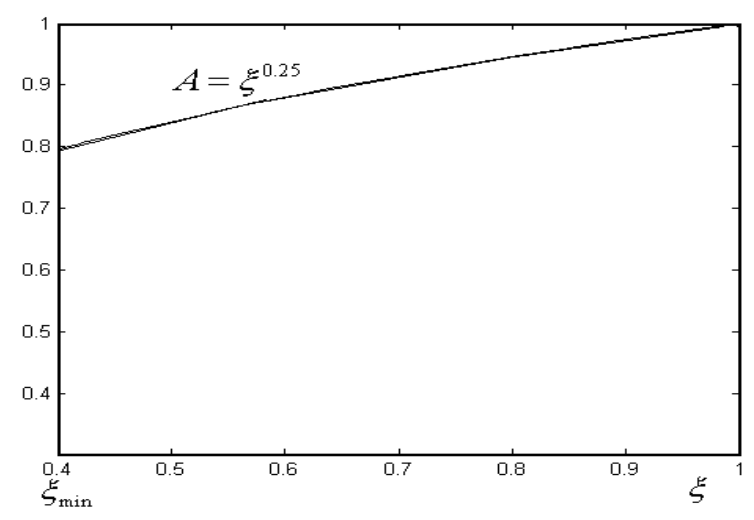

Hình 3. Đồ thị số mũ khuếch đại của [7] (gần tuyến tinh)

Như đã đề cập trong phần I, đối với ảnh tối, thuật toán nâng cao độ tương phản trực tiếp trên không thay đổi được độ sáng của ảnh. Để giải quyết vấn đề thay đổi độ sáng của ảnh sau khi tăng cường độ tương phản (địa phương) chúng tôi đề xuất xây dựng một biến đổi ảnh $\mathrm{F}$ của từng kênh ảnh xám của tổ hợp kênh ảnh đầu vào. Khi đó độ tương phản được tính công thức (13) sẽ được thay đổi thành:

$$
C_{\mathrm{ij}}=\left|\frac{F(\mathrm{i}, \mathrm{j})-\delta_{\mathrm{ij}}(F)}{F(i, j)+\delta_{\mathrm{ij}}(F)}\right|,
$$

ở đây ta đồng nhất biến đổi $\mathrm{F}$ với ảnh $\{\mathrm{F}(\mathrm{i}, \mathrm{j})\}$. 
Trong [6], các tác giả cũng đã xây dựng một biến đổi mờ hóa ảnh áp dụng cho quy trình nâng cao độ tương phản trực tiếp. Như đã phân tích ở phần I, biến đổi ảnh này có thể làm mất chi tiết ảnh. Để chi tiết chúng tôi sẽ tóm lược lại phép mờ hóa kênh ảnh của [7] như mục sau:

\section{II.4. Ước lượng dải động mức xám và biến đổi - mò̀ hóa ảnh sử dụng S-function}

Dựa trên histogram của ảnh, trong $[6,7]$ các tác giả đã nhận thấy đỉnh đầu tiên của histogram của ảnh liên quan đến vùng nền và mức xám lớn hơn đỉnh sau cùng có thể là nhiễu. Vì vậy các tác giả đã đề xuất cách xác định một dải động của mức xám như sau:

$$
\text { Đặt } \frac{\sum_{i=1}^{k} H_{\text {is }}{ }_{\text {max }}\left(g_{i}\right)}{k} \text {, }
$$

trong đó $\mathrm{k}$ là số điểm cực đại địa phương) của histogram, $\operatorname{His}_{\max }\left(\mathrm{g}_{1}\right), \ldots, \operatorname{His}_{\max }\left(\mathrm{g}_{\mathrm{k}}\right)$ là các điểm cực đại địa phương của histogram, $\mathrm{g}_{1}, \mathrm{~g}_{\mathrm{k}}$ là điểm cực đại địa phương đầu tiên và sau cùng tương ứng sao cho:

$\operatorname{His}_{\text {max }}\left(\mathrm{g}_{1}\right) \geq \overline{H i \mathrm{~s}_{\max }(g)}, \operatorname{His}_{\max }\left(\mathrm{g}_{\mathrm{k}}\right) \geq \overline{\operatorname{His}_{\text {max }}(g)}$

Dải động mức xám là đoạn $[\mathrm{a}, \mathrm{c}]$ ở đó

$$
\begin{aligned}
& a=\min \left\{\left(1-f_{2}\right)\left(g_{1}-L_{\min }\right)+L_{\min }, B_{1}\right\} \\
& c=\max \left\{f_{2}\left(L_{\max }-g_{k}\right)+g_{k}, B_{2}\right\},
\end{aligned}
$$

trong đó $\mathrm{B}_{1}, \mathrm{~B}_{2}$ được xác định :

$$
\begin{aligned}
& \sum_{i=L_{\min }}^{B_{1}} H \operatorname{is}(i) \approx f_{1} \sum_{i=L_{\min }}^{L_{\max }} H \operatorname{is}(i) \\
& \sum_{i=B_{2}}^{L_{\max }} H \operatorname{is}(i) \approx f_{1} \sum_{i=L_{\min }}^{L_{\max }} H \operatorname{is}(i),
\end{aligned}
$$

và $\mathrm{L}_{\min }, \mathrm{L}_{\max }$ là giá trị mức xám nhỏ nhất và lớn nhất của kênh ảnh, các hằng số $\mathrm{f}_{1}=0.01, \mathrm{f}_{2}=0.5$ được xác định bằng thực nghiệm.

Từ dải động mức xám $[\mathrm{a}, \mathrm{c}]$ đã ước lượng trên, trong [6] đã trình bày một thuật toán để mờ hóa ảnh đầu vào trước khi nâng cao độ tương phản của ảnh đầu vào.

\section{KỸ THUẬT ĐỀ XUẤT}

Phần này trình bày kỹ thuật ước lượng các dải động của mức xám sử dụng phân cụm mờ $\mathrm{FCM}$, phép biến đổi kênh ảnh và kỹ thuật nâng cao độ tương phản của ảnh màu xét trong biểu diễn màu HSV.

\section{III.1. Ước lượng nhiều dải động mức xám dựa vào phân cụm mò̀ FCM}

Phân cụm mờ c-mean (FCM [4]), được sử dụng hiệu quả trong một số nghiên cứu về nâng cao độ tương phản của ảnh một kênh.

Trong [16] đã trình bày một cách xác định dải động của miền giá trị mức xám bằng cách sử dụng thuật toán phân cụm mờ FCM, khi đó các cụm ảnh có tính chất đều hơn, và do đó việc xác định dải động mức xám là tương đối dễ dàng. Ngoài ra trong [15] các tác giả cũng sử dụng $\mathrm{FCM}$ để phân đoạn histogram và nâng cao độ tương phản theo bộ phận của histogram.

Để có thể ước lượng tự động dải động mức xám cho nhiều loại ảnh khác nhau như ảnh tối, ảnh sáng, ảnh có độ tương phản thấp và ảnh có độ tương phản cao, chúng tôi đề xuất sử dụng phân cụm mờ để ước lượng dải động của mức xám của từng kênh ảnh của ảnh đa kênh. Lưu ý rằng trong một số biểu diễn màu như biểu diễn màu RGB, các kênh ảnh là không độc lập mà có độ tương quan cao, vì thế cách làm ước lượng dải động của từng kênh ảnh độc lập là không hoàn toàn phù hợp trong trường hợp tổng quát. Trong kỹ thuật của chúng tôi, do đặc tính của thuật toán phân cụm mờ là thích hợp cho dữ liệu đầu vào dạng vector số nên kỹ thuật sử dụng FCM sẽ có tính khái quát cao khi áp dụng cho các kênh ảnh có sự tương quan.

Sau khi phân cụm, việc ước lượng dải động mức xám của từng cụm sẽ dễ dàng hơn do tính đồng nhất cao của giá trị mức xám trong một cụm.

Với một tổ hợp K kênh ảnh của ảnh I (trong một biểu diễn màu), để thuận tiện chúng ta ký hiệu $I_{\overline{1, K}}=\left\{\mathrm{I}_{1}, \mathrm{I}_{2}, \ldots, \mathrm{I}_{\mathrm{K}}\right\}$, sử dụng thuật toán phân cụm mờ $\mathrm{FCM}$ phân cụm $I_{\overline{1, K}}$ thành $\mathrm{C}$ cụm, $\mathrm{C} \geq 2$. Thuật toán lặp FCM cực tiểu hóa hàm mục tiêu:

$$
J(V, \mu)=\sum_{i, j} \sum_{c=1}^{c} \mu_{i, j, c}^{2}\left\|I_{\overline{1, K}}(i, j)-V_{c}\right\|^{2} \rightarrow \min
$$


với độ đo khoảng cách Ơcơlit, $\left\|I_{\overline{1, K}}(i, j)-V_{c}\right\|^{2}=$ $\sum_{k=1}^{K}\left(I_{k}(i, j)-V_{c}(k)\right)^{2}$ và các ràng buộc biến như sau:

(i) $\mu_{i, j, c} \in[0,1], 1 \leq c \leq C$

(ii) $\sum_{\mathrm{c}=1}^{\mathrm{C}} \mu_{\mathrm{i}, \mathrm{j}, \mathrm{c}}=1, \forall 1 \leq \mathrm{i} \leq \mathrm{M}, 1 \leq \mathrm{j} \leq \mathrm{N}$

(iii) $\sum_{\mathrm{i}, \mathrm{j}} \mu_{\mathrm{i}, \mathrm{j}, \mathrm{c}}>0, \forall 1 \leq \mathrm{c} \leq \mathrm{C}$

Như vậy với FCM chúng ta nhận được bảng các giá trị độ thuộc từng cụm cho từng điểm ảnh là $\left\{\mu_{i, j, c}\right\}$, trong đó $1 \leq \mathrm{c} \leq \mathrm{C}, 1 \leq \mathrm{i} \leq \mathrm{M}$ và $1 \leq \mathrm{j} \leq \mathrm{N}$.

Định nghĩa 3.1: Histogram mờ:

Giả sử $\left\{\mu_{i, j, c}\right\}$ là bảng độ thuộc thỏa mãn ở công thức (24), histogram mờ theo từng kênh $\mathrm{I}_{\mathrm{k}}$ của ảnh $\mathrm{I}$ (trong một biểu diễn màu), $1 \leq \mathrm{k} \leq \mathrm{K}$, ký hiệu là $h_{c}^{k}$ được xác định như sau:

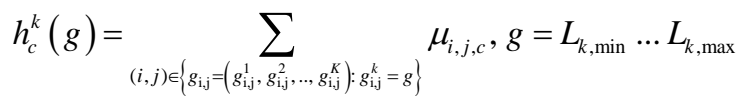

Nhận xét: $K h i \mathrm{~K}=1, \mathrm{C}=\mathrm{L}_{\max }-\mathrm{L}_{\min }+1$ và $\forall c=\overline{1, L_{1, \max }-L_{1, \min }+1}, \mu_{i, j, c}=\left\{\begin{array}{l}1, I_{1}(i, j)=c-1 \\ 0\end{array}\right.$ thì $\left\{h_{c}^{1}(c-1)\right\}_{c=\overline{1, L_{k, k n a x}, \mathrm{~L}_{k, \min }+1}}$ trùng với histogram thông thường của ảnh xám.

Thuật toán 1. Ước lượng $\mathbf{C}$ dải động mức xám của một cụm trong một tổ hợp kênh ảnh sử dụng histogram mờ.

Đầu vào: $\mathrm{K}$ kênh của ảnh $\mathrm{I}$ (trong một biểu diễn màu), $I_{\overline{1, K}}=\left\{\mathrm{I}_{1}, \mathrm{I}_{2}, \ldots, \mathrm{I}_{\mathrm{K}}\right\}$, tham số $\mathrm{C} \in N^{+}, C \geq 2$, ngưỡng $\mathrm{f}_{\text {cut }}\left(\mathrm{f}_{\text {cut }}>0\right.$, đủ nhỏ), $\mathrm{M} \times \mathrm{N}$ là kích thước của ảnh I.

Đầu ra: $\left\{B_{k, 1, c}, B_{k, 2, c}\right\}_{k=\overline{1, K}, c=\overline{1, C}}$, trong đó $L_{k, \text { min }} \leq B_{k, 1, c}<B_{k, 2, c} \leq L_{k, \max }, \forall c=\overline{1, C}, k=\overline{1, K}$
Bước 1: Phân $\mathrm{C}$ cụm tập vector dữ liệu $\left\{I_{k}(i, j)\right\}_{i=\overline{1, M}, j=\overline{1, N}, k=\overline{1, K}}$ bằng thuật toán $\mathrm{FCM}$ chuẩn ta được $\left\{V_{c}\right\}_{c=1}^{C},\left\{\mu_{i, j, c}\right\}_{i=\overline{1, M}, j=\overline{1, N}, c=\overline{1, C}}$, theo công thức (24).

Bước 2: Xác định histogram mờ $\left\{h_{c}^{k}\right\}_{c=\overline{1, C}, k=\overline{1, K}}$ theo công thức (25).

Bước 3: $\forall k=\overline{1, K}, c=\overline{1, C}$

$B_{k, 1, c}=\underset{B \in\left[\mathbb{L}_{k, \text { min }}, \mathbf{L}_{k, \text { max }}\right.}{\arg \min }\left\{\sum_{g=L_{k, \text { min }}}^{B} h_{c}^{k}(g)>f_{\text {cut }} \sum_{g=L_{k, \text {,min }}}^{L_{k, \text { max }}} h_{c}^{k}(g)\right\}$

$B_{k, 2, c}=\underset{B \in\left[\mathbb{B}_{k, 1, c}+1, L_{k, \text { max }}\right]}{\arg \min }\left\{\sum_{g=B}^{L_{k, \text { max }}} h_{c}^{k}(g)<f_{c u t} \sum_{g=L_{k, \text { min }}}^{L_{k, \text { max }}} h_{c}^{k}(g)\right\}$

Trả về: $\left\{\boldsymbol{B}_{k, 1, c}, \boldsymbol{B}_{k, 2, c}\right\}_{k=\overline{1, K}, c=\overline{1, C}}$

Thuật toán 1 có độ phức tạp tồi nhất là $\mathrm{O}\left(\mathrm{M}^{*} \mathrm{~N}^{*} \mathrm{~L}\right)$, trong đó $L$ là tham số số lần lặp tối đa của thuật toán FCM chuẩn.

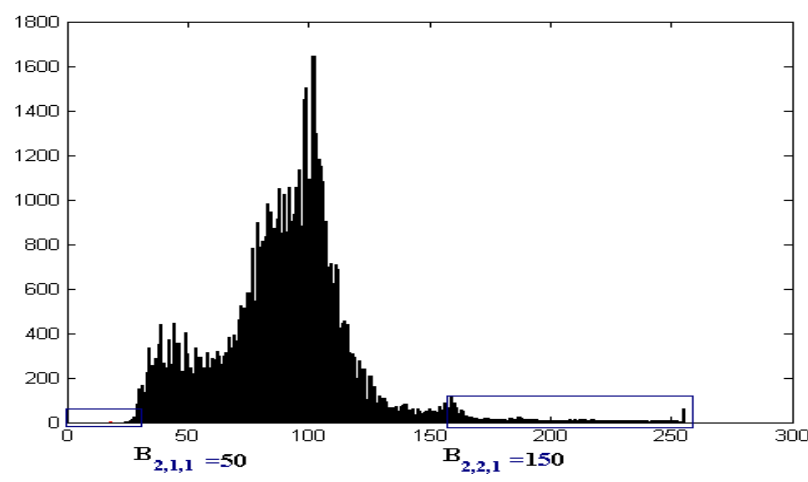

Hình 4. Histogram của kênh $V$, cụm số 1 ảnh \#4 (C= 5), trục hoành biểu diễn giá trị xám, trục tung biểu thị tần suất

\section{III.2. Biến đổi kênh ảnh}

Định nghĩa 3.2: Phép biến đổi kênh ảnh của một tổ hợp kênh trong một biểu diễn màu của ảnh đầu vào.

Xét $\mathrm{K}$ kênh của ảnh $\mathrm{I}, I_{\overline{1, K}}=\left\{\mathrm{I}_{1}, \mathrm{I}_{2}, \ldots, \mathrm{I}_{\mathrm{K}}\right\}$ trong một biểu diễn màu, $\mathrm{C} \in N^{+}, C \geq 2$ là số cụm, $\left\{\boldsymbol{B}_{k, 1, c}, \boldsymbol{B}_{k, 2, c}\right\}_{k=\overline{1, K}, c=\overline{1, C}}$ là các dải động mức xám được xác định nhờ thuật toán 1 . 
Với mỗi $k=\overline{1, K}$, chúng ta xác định một biến đổi $F_{k}$ cho kênh ảnh $I_{k}$ như sau:

$$
F_{k}(i, j)=\left[L_{k, \min }+\frac{\left(L_{k, \text { max }}-L_{k, \min }\right) \sum_{c=1}^{c} \operatorname{clip}\left(\frac{I_{k}(i, j)-B_{k, 1, c}}{B_{k, 2, c}-B_{k, 1, c}}\right)}{C}\right],
$$

trong đó $k=\overline{1, K}, i=\overline{1, M}, j=\overline{1, N}, \operatorname{clip}(\mathrm{x})=$ $\min \{\max \{\mathrm{x}, 0\}, 1\}$ và $[\mathrm{x}]$ chỉ phần nguyên của số thực $\mathrm{x}$.

Nhận xét: Biến đổi ảnh $\mathrm{F}_{\mathrm{k}}$ bảo toàn thứ tự, nghĩa là

$$
I_{k}(i, j) \leq I_{k}\left(i^{\prime}, j^{\prime}\right) \Rightarrow L_{k, \min } \leq F_{k}(i, j) \leq F_{k}\left(i^{\prime}, j^{\prime}\right) \leq L_{k, \max }
$$

Kỹ thuật nâng cao độ tương phản ảnh màu trong biểu diễn màu HSV, ảnh RGB đầu vào được chuyển sang biểu diễn HSV. Sau đó, thuật toán sẽ sử dụng ảnh 2 kênh $\{\mathrm{S}, \mathrm{V}\}$ như một ảnh 2 kênh đầu vào để thực hiện phép nâng cao độ tương phản được thực hiện theo thuật toán 2 như dưới đây. Hai kênh ảnh $\{S$, V\} mới là đầu ra thu nhận được sẽ kết hợp với kênh $\mathrm{H}$ gốc của ảnh đầu vào để biến đổi ngược từ biểu diễn màu HSV trở về biểu diễn màu RGB. Đầu ra của biến đổi ngược HSV sang RGB là ảnh kết quả cuối cùng được trả về. Thuật toán 2 được thực hiện như sau:

Thuật toán 2. Nâng cao độ tương phản ảnh màu sử dụng biểu diễn màu HSV.

Đầu vào: Ảnh màu I trong biểu diễn màu $R G B$, có kích thước $\mathrm{M} \times \mathrm{N}$. Tham số $\mathrm{C} \in N^{+}, C \geq 2$, ngưỡng $\mathrm{f}_{\text {cut }}\left(\mathrm{f}_{\text {cut }}>0\right.$, đủ nhỏ $), \mathrm{d}(\mathrm{d} \times \mathrm{d}$ là kích thước cửa sổ $)$.

Đầu ra: Ảnh màu RGB $\mathrm{I}_{\text {new }}$, và tùy chọn trả về:

Giá trị tương phản trung bình $\mathrm{CM}_{\mathrm{R}}, \mathrm{CM}_{\mathrm{G}}, \mathrm{CM}_{\mathrm{B}}$

Giá trị $\mathrm{E}_{\text {avg }}, \mathrm{H}_{\text {avg }}$

Bước 1: Gọi $\left(\mathrm{I}_{\mathrm{H}}, \mathrm{I}_{\mathrm{S}}, \mathrm{I}_{\mathrm{V}}\right)$ là biểu diễn màu của $\mathrm{I}$ trong không gian màu HSV. Lượng hóa để coi các kênh $\mathrm{I}_{\mathrm{S}}$, $\mathrm{I}_{\mathrm{V}}$ như là các ảnh đa cấp xám.

Bước 2: Với dữ liệu đầu vào là tổ hợp kênh $\left(\mathrm{I}_{S}, \mathrm{I}_{\mathrm{V}}\right)$, tham số số cụm là $\mathrm{C}$ và ngưỡng $\mathrm{f}_{\text {cut }}$, gọi thuật toán 1 để ước lượng các dải động mức xám theo kênh $\mathrm{I}_{\mathrm{S}}, \mathrm{I}_{\mathrm{V}}$. (xem công thức (24), (25) và (26))
Bước 3: Xác định ảnh biến đổi $\mathrm{F}_{\mathrm{S}}, \mathrm{F}_{\mathrm{V}}$ của kênh $\mathrm{I}_{\mathrm{S}}, \mathrm{I}_{\mathrm{V}}$ tương ứng theo công thức (27), định nghĩa 3.2 với các dải động mức xám được ước lượng từ bước 2 cho mỗi kênh $S$ và kênh $V$.

Bước 4: Tính tham số của [7] cho kênh $\mathrm{F}_{\mathrm{S}}, \mathrm{F}_{\mathrm{V}}$ như đã trình bày trong mục 2 (xem các công thức từ (6) đến (12) với kích thước cửa sổ $\mathrm{dxd}$, cụ thể là các giá trị mức xám không thuần nhất $\left\{\delta_{\mathrm{S}, \mathrm{ij}}\right\},\left\{\delta_{\mathrm{V}, \mathrm{ij}}\right\}$, số mũ khuếch đại $\left\{\xi_{\mathrm{S}, \mathrm{ij}}\right\},\left\{\xi_{\mathrm{V}, \mathrm{ij}}\right\}$ tại từng điểm ảnh của kênh $F_{\mathrm{S}}$ và kênh $\mathrm{F}_{\mathrm{V}}$.

Bước 5: Tính độ tương phản và xác định kênh ảnh xám mới của kênh $\mathrm{F}_{\mathrm{S}}$ và kênh $\mathrm{F}_{V}$, $F_{S} \mapsto I_{S, \text { new }}, F_{V} \mapsto I_{V, \text { new }}$ như sau:

Với kênh $\mathrm{F}_{\mathrm{S}}$ và kênh $\mathrm{F}_{\mathrm{V}}$ : Tính độ tương phản

$C_{\mathrm{S}, \mathrm{ij}}=\left|\frac{F_{S}(i, j)-\delta_{\mathrm{ij}}\left(F_{S}\right)}{F_{S}(i, j)+\delta_{\mathrm{ij}}\left(F_{S}\right)}\right|, C_{\mathrm{V}, \mathrm{ij}}=\left|\frac{F_{V}(i, j)-\delta_{\mathrm{ij}}\left(F_{V}\right)}{F_{V}(i, j)+\delta_{\mathrm{ij}}\left(F_{V}\right)}\right|$

Tính giá trị mức xám mới của kênh $\mathrm{S}$ và $\mathrm{V}$ :

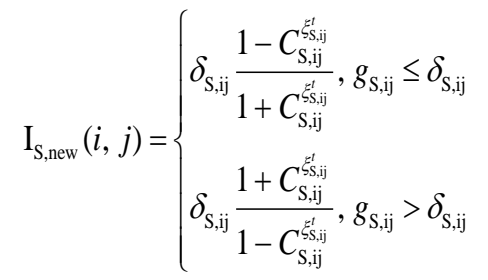

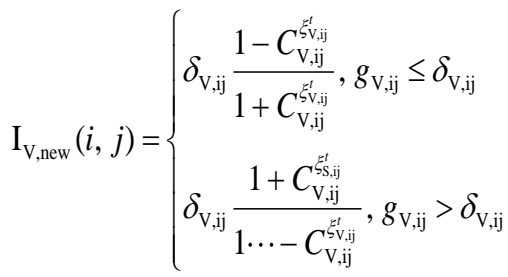

Lưu ý ở đây kênh $\mathrm{S}$ được đánh chỉ số $\mathrm{k}=1$, kênh $\mathrm{V}$ được đánh chỉ số $\mathrm{k}=2$.

Bước 6: Chuyển đổi ảnh $\left(\mathrm{I}_{\mathrm{H}}, \mathrm{I}_{\mathrm{S} \text {,new }}, \mathrm{I}_{\mathrm{V} \text {,new }}\right)$ trong biểu diễn màu HSV về biểu diễn màu RGB, ta được ảnh $\mathrm{I}_{\text {new. }}$.

Bước 7: Bước tùy chọn, tính các chỉ số khách quan $\mathrm{CM}_{\{\mathrm{R}, \mathrm{G}, \mathrm{B}\}}, \mathrm{E}_{\mathrm{avg}}$ và $\mathrm{H}_{\mathrm{avg}}$

7.1: Tính tham số của [7] cho kênh $\mathrm{I}_{R}, \mathrm{I}_{\mathrm{G}}$ và $\mathrm{I}_{\mathrm{B}}$ của ảnh gốc I như đã trình bày trong mục II.3 (xem các công thức từ (6) đến (12) với kích thước cửa sổ $\mathrm{dxd}$, cụ thể là các giá trị mức xám không thuần nhất $\left\{\delta_{\mathrm{R}, \mathrm{ij}}\right\},\left\{\delta_{\mathrm{G}, \mathrm{ij}}\right\}$, và $\left\{\delta_{\mathrm{B}, \mathrm{ij}}\right\}$ của kênh $\mathrm{I}_{\mathrm{R}}, \mathrm{I}_{\mathrm{G}}$ và $\mathrm{I}_{\mathrm{B}}$ tương ứng. 
7.2: Tính $\mathrm{CM}_{\mathrm{R}}, \mathrm{CM}_{\mathrm{G}}, \mathrm{CM}_{\mathrm{B}}$ theo công thức (31), cụ thể là:

$$
\begin{gathered}
C M_{R}=\frac{\sum_{\mathrm{ij}} \frac{\left|I_{n e w, R}(i, j)-\delta_{R, \mathrm{ij}}\right|}{I_{n e w, R}(i, j)+\delta_{R, \mathrm{jj}}}}{M N} \\
C M_{G}=\frac{\sum_{\mathrm{ij}} \frac{\left|I_{n e w, G}(i, j)-\delta_{G, \mathrm{ij}}\right|}{I_{n e w, G}(i, j)+\delta_{G, \mathrm{ij}}}}{M N} \\
C M_{B}=\frac{\mid \frac{\left|I_{\text {new }, B}(i, j)-\delta_{B, \mathrm{ij}}\right|}{I_{\text {new }, B}(i, j)+\delta_{B, \mathrm{ij}}}}{M N}
\end{gathered}
$$

7.3: Tính $\mathrm{E}_{\text {avg }}=\mathrm{E}_{\text {avg }}\left\{\mathrm{I}_{\text {new, }, \mathrm{R}}, \mathrm{I}_{\text {new,G }}, \mathrm{I}_{\text {new,B } \mathrm{B}}\right\}$ theo công thức (32). Tính $\mathrm{H}_{\text {avg }}=\mathrm{H}_{\text {avg }}\left\{\mathrm{I}_{\text {new }, \mathrm{R}}, \mathrm{I}_{\text {new,G }}, \mathrm{I}_{\text {new }, \mathrm{B}}\right\}$ theo công thức (35).

Trả về: $\mathrm{I}_{\text {new }}$, và các tùy chọn được trả về

$$
\begin{aligned}
& \mathrm{CM}_{\mathrm{R}}, \mathrm{CM}_{\mathrm{G}}, \mathrm{CM}_{\mathrm{B}}, \\
& \mathrm{E}_{\text {avg }}, \mathrm{H}_{\text {avg. }} .
\end{aligned}
$$

Thuật toán 2 có độ phức tạp tương đương thuật toán gốc [7].

Mô hình kiến trúc hệ thống đề xuất (Hình 5) được xây dựng và tương tác như sau:

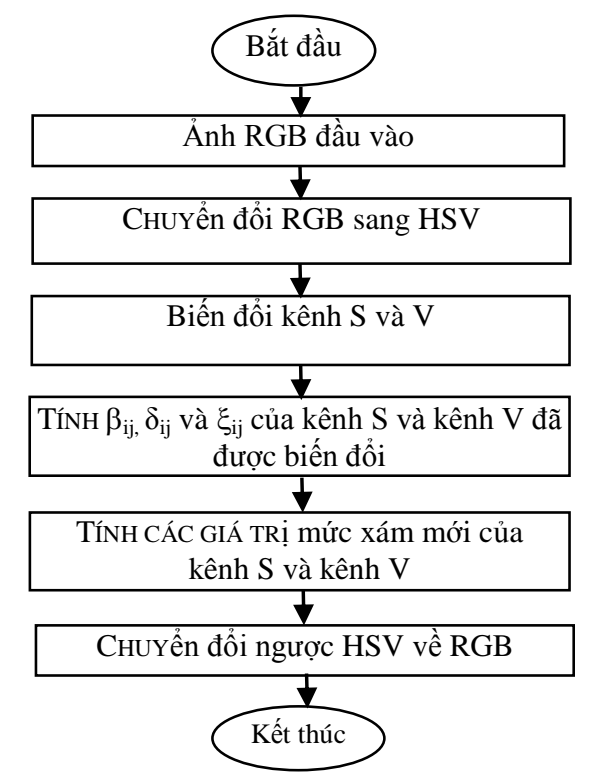

Hình 5. Lưu đồ xử lý của các thuật toán đề xuất.

\section{THỰC NGHIÊM}

\section{IV.1. Ảnh thử nghiệm}

Để đánh giá hiệu quả của phương pháp được đề xuất, chúng tôi sẽ đưa ra một số thực nghiệm cơ bản và so sánh kết quả với kết quả của phương pháp được mô tả trong [7]. Trong thực nghiệm chúng tôi đã sử dụng đa dạng các kiểu ảnh màu, các kênh màu của ảnh vệ tinh. Các ảnh đa màu được chọn điển hình từ loại ảnh tối, ảnh sáng, ảnh có độ tuoong phản các kênh là thấp, ảnh có độ tương phản các kênh là cao v.v... Các ảnh có thể có độ sáng thấp và chi tiết ảnh là không quan sát được rõ bằng mắt.

Việc lựa chọn ảnh thử nghiệm đa dạng như vậy nên chúng tôi tin tưởng rằng việc kiểm thử các thuật toán của chúng tôi sẽ cho một đánh giá khách quan về hiệu quả của chúng.

Tập ảnh màu (đánh số \#1 - \#6) được dùng để thể hiện trong khuôn khổ bài báo này thu nhận từ tập ảnh RGB được công bố trong [14] (ảnh từ \#3 đến \#5 trong hình 6), ảnh vệ tinh huyện Lạc Thủy của Việt Nam (ảnh \#6 trong Hình 6).

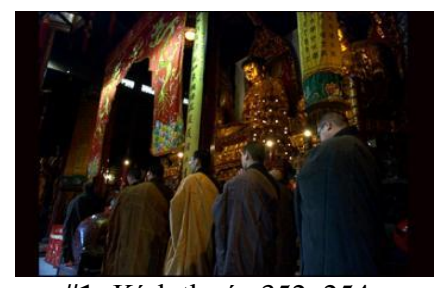

\#1: Kích thước 352x254

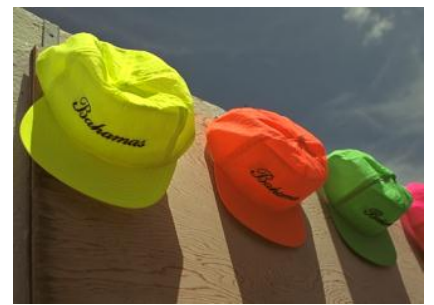

\#3: Kích thước 512x384

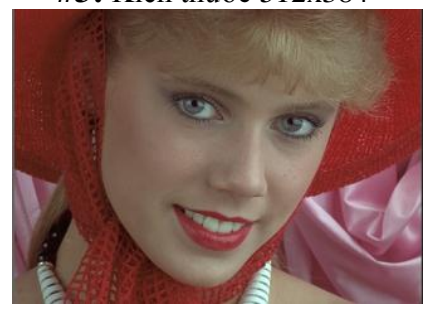

\#5: Kích thước 512×384 Hình 6. Một số ảnh gốc được dùng cho thư nghiệm. 


\section{IV.2. Tính độ thuần nhất kênh ảnh}

Chất lượng ảnh được nâng cao độ tương phản phụ thuộc vào giá trị thuần nhất tại mỗi điểm ảnh, bởi vì độ đo thuần nhất liên quan chính đến các thông tin địa phương của một ảnh và phản ánh tính đều của các vùng ảnh và nó đóng vai trò quan trọng trong nâng cao chất lượng ảnh [7].

Trong thử nghiệm các giá trị địa phương được mô tả trong [7] được tính với kích thước cửa số $3 \times 3$, đặc biệt công thức (11) tính các giá trị thuần nhất

$$
H O_{\mathrm{ij}}=\overline{E_{\mathrm{ij}}} * \overline{V_{i j}} * \overline{H_{\mathrm{ij}}} * \overline{R_{4, \mathrm{j}}}=\left(1-E_{\mathrm{ij}}\right) *\left(1-V_{\mathrm{ij}}\right) *\left(1-H_{\mathrm{ij}}\right) *\left(1-R_{4, \mathrm{ij}}\right)
$$

được chúng tôi thay thế bởi công thức sau:

$$
H O_{\mathrm{ij}}=\max \left\{\overline{E_{\mathrm{ij}}} * \overline{H_{\mathrm{ij}}}, \overline{V_{\mathrm{ij}}} * \overline{R_{4, \mathrm{j}}}\right\},
$$

ở đây cần nhấn mạnh là các giá trị entropy địa phương $\mathrm{H}_{\mathrm{ij}}$ rất nhạy với nhiễu và sự thay đổi của giá trị mức xám, nên khi kết hợp theo công thức (11) có thể tạo ra giá trị độ thuần nhất rất không trơn và do đó ảnh hưởng đến độ trơn của ảnh nâng cao độ tương phản đầu ra. Để đi tới đề xuất công thức đơn giản (30) chúng tôi đã khảo sát trên 30 công thức khác nhau kết hợp 4 đặc trưng địa phương $\mathrm{E}_{\mathrm{ij}}, \mathrm{H}_{\mathrm{ij}}, \mathrm{V}_{\mathrm{ij}}, \mathrm{R}_{4, \mathrm{ij}}$, trong đó đã sử dụng các toán tử kết nhập mờ thông dụng như min, max, product, Yager, Zimmerman, Hamacher, Dombi, Aczel, tổng trọng số v.v... [10] và thấy rằng công thức (30) là phù hợp cho đa dạng ảnh màu RGB (các ảnh đã được nâng cao độ tương phản khi sử dụng công thức (30) và thuật toán của [7] đều trơn). Ngoài ra, trong [7] các tác giả cũng đã chỉ sử dụng $\boldsymbol{E}_{i j}$ và $\boldsymbol{H}_{i j}$ để tính giá trị trung bình không thuần nhất của điểm ảnh khi nâng cao độ tương phản của kênh ảnh theo phương pháp trực tiếp.

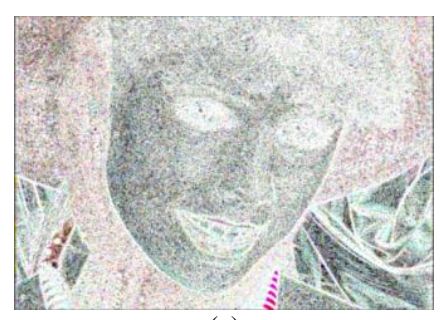

(a)

Hình 7. $\left\{H_{i j}\right\} \# 5 .(a)\left\{V_{i j}\right\}$; (b) 3 kênh $R, G$ và $B$ với ảnh \#5.

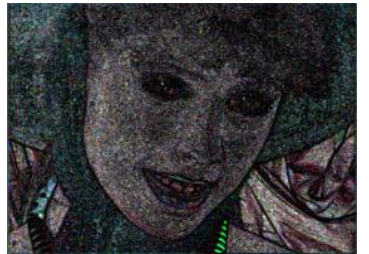

(a)

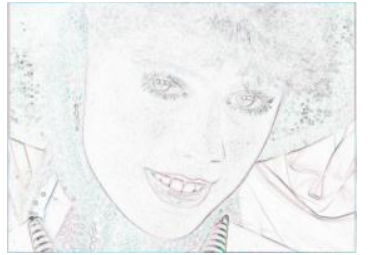

(c)

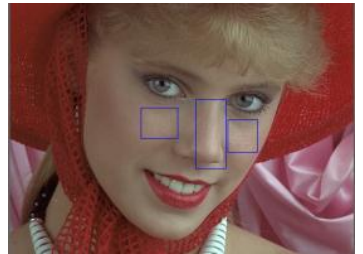

(b)

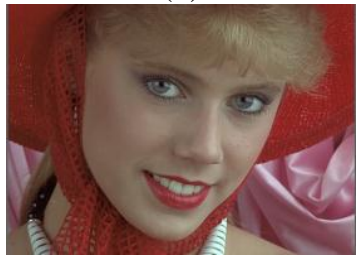

(d)
Hình 8. Độ thuần nhất tính theo công thức gốc (11) [7] (a) Anh nâng cao độ tương phản 3 kênh $R, G$ và $B$ sủ dụng công thức (11) (b) Độ thuần nhất tính theo công thức (30) (c) Anh nâng cao sư dụng công thức (30) (d)

Hình 7.a chứng tỏ các giá trị độ thuần nhất tại các điểm ảnh của ảnh \#5 và ảnh kết quả của phép nâng cao của [7] khi dùng công thức gốc (11) là không đủ trơn (xem hình 7.b ở các vùng đánh dấu ô chữ nhật). Ngoài ra thử nghiệm với các ảnh màu khác nhau cùng thuật toán của $[6,7]$ cho từng kênh ảnh $R, G$ và $B$ chúng tôi cũng nhận thấy cả 2 công thức (11) và công thức (30) cùng cho ảnh đầu ra của thuật toán [7] có sự tương phản mạnh ở vùng có độ thuần nhất thấp (chẳng hạn, vùng đôi mắt, xem hình $8 . b$ và $8 . d$ ) và mức độ sáng của hình ảnh được nâng cao là như nhau.

\section{IV.3. Đánh giá thuật toán 2}

Khi đánh giá kết quả thuật toán 1 và thuật toán 2 của chúng tôi so với thuật toán gốc được công bố trong [7], vì [7] chỉ được phát biểu ứng dụng cho ảnh đa cấp xám, trong khi thuật toán của chúng tôi được phát biểu cho ảnh màu để đảm bảo tính khách quan ngoài đánh giá bằng trực quan chúng tôi sẽ sử dụng các chỉ số khách quan để đánh giá. Như vậy cách đánh giá của chúng tôi là:

- Bằng trực quan.

- Sử dụng chỉ số biểu diễn độ tương phản trực tiếp.

- Chỉ số đánh giá khách quan khác.

Lưu ý rằng trong các thực nghiệm của thuật toán gốc $[7,6]$ các tác giả của các thuật toán gốc chỉ sử 
dụng đánh giá bằng cảm nhận trực quan, không dùng bất kỳ một chỉ số khách quan nào để đánh giá.

Các chỉ số đánh giá khách quan độ tương phản ảnh được dùng trong bài báo này cụ thể là:

(1) Chỉ số độ tương phản trực tiếp được lấy trung bình trên toàn bộ các điểm ảnh của một kênh ảnh $\mathrm{I}_{\mathrm{k}}$, so với một kênh ảnh gốc $\mathrm{I}_{\mathrm{k}}\left(\mathrm{I}_{\mathrm{k}}\right.$, và $\mathrm{I}_{\mathrm{k}}$ có cùng kích thước $\mathrm{M} \times \mathrm{N}$ ), chúng được cho như sau:

$$
C M\left(I_{k}, I_{k}^{\prime}\right)=\frac{\sum_{\mathrm{i}, \mathrm{j}} \frac{\left|I_{k}^{\prime}(i, j)-\delta_{k, \mathrm{j} j}\right|}{I_{k}^{\prime}(i, j)+\delta_{k, \mathrm{ij}}}}{M N},
$$

ở đây $\delta_{\mathrm{k}, \mathrm{ij}}$ là giá trị mức xám không thuần nhất tại điểm ảnh $(i, j)$ của $\mathrm{I}_{\mathrm{k}}$ (xem ký hiệu ở bảng 1 , và xem [7]).

Nhận xét: Nói chung ta có,

$$
\text { - } C M\left(I_{k}, I_{k}^{\prime}\right) \neq C M\left(I_{k}^{\prime}, I_{k}\right)
$$

$$
C M\left(J_{k}, I_{k}^{\prime \prime}\right)>C M\left(J_{k}, I_{k}^{\prime}\right) \nRightarrow C M\left(I_{k}, I_{k}^{\prime \prime}\right)>C M\left(I_{k}, I_{k}^{\prime}\right)
$$

ở đây $\mathrm{I}_{\mathrm{k}}, \mathrm{J}_{\mathrm{k}}, \mathrm{I}_{\mathrm{k}}$, và $\mathrm{I}_{\mathrm{k}}$, là các kênh ảnh có cùng kích thước. Nhận xét nhấn mạnh là phép nâng cao độ tương phản của ảnh biến đổi từ ảnh gốc thì chưa chắc đã là phép nâng cao độ tương phản của ảnh gốc.

(2) Chỉ số entropy được lấy trung bình trên toàn bộ các điểm ảnh và các kênh ảnh, chúng được cho như sau:

$$
\begin{gathered}
E\left(I_{k}\right)=-\sum_{g=L \min _{k}}^{L \max _{k}} p_{k}(g) \log _{2}\left(p_{k}(g)\right) \\
E_{\text {avg }}\left(I_{\overline{1, K}}\right)=\frac{\sum_{k=1}^{K} E\left(I_{k}\right)}{K},
\end{gathered}
$$

trong đó $p_{k}(g) \stackrel{\text { def }}{=} \frac{\#\left\{I_{k}(i, j)=g\right\}}{M N}$ và quy ước $0 * \log _{2}(0)=0$.

Giá trị của chỉ số entropy cao thì có thể xem ảnh là giầu tính chi tiết. Chỉ số entropy của ảnh kết quả càng cao thì khả năng là ảnh kết quả bảo toàn tốt tính chi tiết của ảnh gốc.

(3) Chỉ số đánh giá khách quan fuzzy-entropy được lấy trung bình trên toàn bộ các điểm ảnh và các kênh ảnh, chúng được cho như sau:
Giả sử $g_{k} \in\left[\mathrm{L}_{\mathrm{k}, \text { min }}, \mathrm{L}_{\mathrm{k}, \text { max }}\right] \mapsto \mu\left(g_{k}\right) \in[0,1]$ là một phép mờ hóa nào đó

$$
H_{\mu}\left(I_{k}\right)=-\sum_{g=L_{\text {min }}}^{L_{\text {max }}}\left(\mu(g) \log _{2}(\mu(g))+(1-\mu(g)) \log _{2}(1-\mu(g))\right) * p_{k}(g)
$$

Dưới đây chúng ta sẽ dùng phép mờ hóa tự nhiên:

$$
g \mapsto \mu(g) \stackrel{\text { def }}{=} \frac{g-L_{k, \min }}{L_{k, \text { max }}-L_{k, \text { min }}}
$$

Khi đó ta viết gọn $\mathrm{H}$ thay cho $\mathrm{H}_{\mu}$, và ta có một độ đo fuzzy-entropy trung bình của K kênh ảnh như sau:

$$
H_{\text {avg }}\left(I_{\overline{1, K}}\right)=\frac{\sum_{k=1}^{K} H\left(I_{k}\right)}{K}
$$

Nhận xét: Giá trị fuzzy entropy càng thấp thì độ phân biệt một điểm ảnh của một kênh ảnh là sáng hoặc tối càng cao tức là ảnh càng có độ tương phản tối - sáng cao, các điểm ảnh của kênh ảnh $\mathrm{I}_{\mathrm{k}}$ có mức xám tương phản cao với mức sáng “xám” ở giữa:

$$
\left|I_{k}(i, j)-\frac{L_{k, \text { min}}+L_{k, \text { max }}}{2}\right| \uparrow \Rightarrow H\left(I_{k}\right) \downarrow
$$

Các ảnh thể hiện trong bài báo này được đánh chỉ số như trong Hình 6.

Trong các nghiên cứu về độ tương phản ảnh như [12], chỉ số khách quan $\gamma$ như sau thường hay được sử dụng:

$$
\gamma_{\mu}\left(I_{k}\right)=-\sum_{g=L_{k, \text { min }}}^{L_{k \text { max }}} \min (\mu(g), 1-\mu(g)) * p_{k}(g)
$$

Thực ra chỉ số $\gamma$ cũng tương tự như chỉ số fuzzy entropy theo ý nghĩa :

$$
\left|I_{k}(i, j)-\frac{L_{k, \text { min }}+L_{k, \text { max }}}{2}\right| \uparrow \Rightarrow \gamma\left(I_{k}\right) \downarrow
$$

Vì vậy trong phần thực nghiệm này, chúng tôi không đề cập đến chỉ số khách quan $\gamma$ khi đánh giá kết quả thực nghiệm.

Với các ảnh thử nghiệm bước đầu tiên là tính các giá trị mức xám không thuần nhất $\left\{\delta_{\mathrm{R}, \mathrm{ij}}\right\},\left\{\delta_{\mathrm{G}, \mathrm{ij}}\right\}$, và $\left\{\delta_{\mathrm{B}, \mathrm{ij}}\right\}$ của 3 kênh $\mathrm{R}, \mathrm{G}$ và $\mathrm{B}$ tương ứng. Các giá trị này được dùng để tính độ đo tương phản $\mathrm{CM}$ trên từng kênh $R, G$ và $B$ của ảnh đầu vào và ảnh kết quả. Từ đó cho chúng ta đánh giá hiệu quả của các thuật toán tăng độ tương phản trực tiếp của ảnh màu RGB. 
Để dễ theo dõi, chúng tôi trình bày lại thuật toán [7] như một thủ tục, giải mã được cho như sau:

Thủ tục 1. Nâng cao độ tương phản ảnh màu trong biểu diễn màu HSV, sử dụng phương pháp của [7] cho từng kênh $\mathrm{S}$ và kênh $\mathrm{V}$.

Đầu vào: Ảnh màu $\mathrm{I}$ trong biểu diễn màu $\mathrm{RGB}$, có kích thước $\mathrm{M} \times \mathrm{N}$. Tham số $\mathrm{d}(\mathrm{d} \times \mathrm{d}$ là kích thước cửa sổ).

Đầu ra: Ảnh màu RGB $\mathrm{I}_{\text {new }}$, và tùy chọn trả về:

Giá trị tương phản trung bình $\mathrm{CM}_{\mathrm{R}}, \mathrm{CM}_{\mathrm{G}}, \mathrm{CM}_{\mathrm{B}}$

Giá trị $\mathrm{E}_{\text {avg }}, \mathrm{H}_{\text {avg }}$

Bước 1: Gọi $\left(\mathrm{I}_{\mathrm{H}}, \mathrm{I}_{\mathrm{S}}, \mathrm{I}_{\mathrm{V}}\right)$ là biểu diễn màu của $\mathrm{I}$ trong không gian màu $\mathrm{HSV}$. Lượng hóa coi các kênh $\mathrm{I}_{\mathrm{S}}, \mathrm{I}_{\mathrm{V}}$ là các ảnh đa cấp xám.

Bước 2: Tính tham số của [7] cho ảnh đa cấp xám $\mathrm{I}_{\mathrm{S}}, \mathrm{I}_{\mathrm{V}}$ như đã trình bày trong mục II.3 (xem các công thức từ (6) đến (12) với kích thước cửa sổ $\mathrm{dxd}$, cụ thể là các giá trị mức xám không thuần nhất $\left\{\delta_{\mathrm{S}, \mathrm{ij}}\right\},\left\{\delta_{\mathrm{V}, \mathrm{ij}}\right\}$, số mũ khuếch đại $\left\{\xi_{\mathrm{S}, \mathrm{ij}}\right\},\left\{\xi_{\mathrm{V}, \mathrm{ij}}\right\}$ tại từng điểm ảnh của ảnh đa cấp xám $\mathrm{I}_{\mathrm{S}}$ và $\mathrm{I}_{\mathrm{V}}$ tương ứng.

Bước 3: Tính độ tương phản và tính mức xám mới của kênh $\mathrm{S}$ và kênh $\mathrm{V}, I_{S} \mapsto I_{S \text {,new }}, I_{V} \mapsto I_{V \text {, new }}$

Với kênh $\mathrm{S}$ và kênh $\mathrm{V}$ : Tính độ tương phản

$$
C_{\mathrm{S}, \mathrm{ij}}=\left|\frac{I_{S}(i, j)-\delta_{\mathrm{S}, \mathrm{j}}}{I_{S}(i, j)+\delta_{\mathrm{S}, \mathrm{j}}}\right|, C_{\mathrm{V}, \mathrm{ij}}=\left|\frac{I_{V}(i, j)-\delta_{\mathrm{V}, \mathrm{ij}}}{I_{V}(i, j)+\delta_{\mathrm{V}, \mathrm{ij}}}\right|
$$

Tính giá trị mức xám mới của kênh $\mathrm{S}$ và kênh V

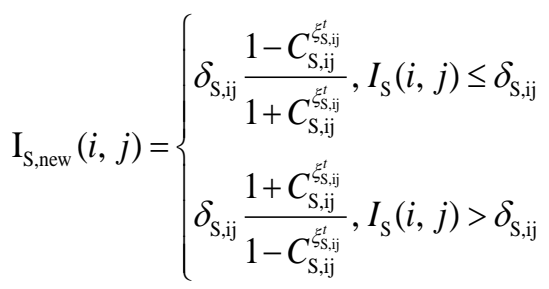

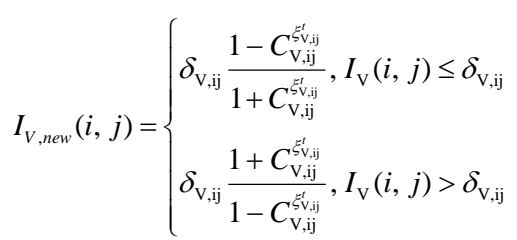

Bước 4: Chuyển đổi ảnh $\left(\mathrm{I}_{\mathrm{H}}, \mathrm{I}_{\mathrm{S} \text {,new }}, \mathrm{I}_{\mathrm{V}, \text { new }}\right)$ trong biểu diễn màu HSV về biểu diễn màu $R G B$, ta được ảnh $\mathrm{I}_{\text {new. }}$.

Bước 5: Bước tùy chọn, tính các chỉ số $\mathrm{CM}_{\{\mathrm{R}, \mathrm{G}, \mathrm{B}\}}$ cho kênh $R, G$ và $B, E_{\text {avg và }} H_{\text {avg }}$ như sau:

5.1: Tính tham số của [7] cho kênh $\mathrm{I}_{R}, \mathrm{I}_{\mathrm{G}}$ và $\mathrm{I}_{B}$ của ảnh gốc I như đã trình bày trong mục II.3 (xem các công thức tử (6) đến (12) với kích thước cửa sổ $\mathrm{dxd}$, cụ thể là các giá trị mức xám không thuần nhất $\left\{\delta_{\mathrm{R}, \mathrm{ij}}\right\}$, $\left\{\delta_{\mathrm{G}, \mathrm{ij}}\right\}$, và $\left\{\delta_{\mathrm{B}, \mathrm{ij}}\right\}$ của các ảnh đa cấp xám $\mathrm{I}_{\mathrm{R}}, \mathrm{I}_{\mathrm{G}}$ và $\mathrm{I}_{\mathrm{B}}$ tương ứng.

5.2: Tính $\mathrm{CM}_{\mathrm{R}}, \mathrm{CM}_{\mathrm{G}}, \mathrm{CM}_{\mathrm{B}}$ theo công thức (31), cụ thể là:

$$
\begin{aligned}
& C M_{R}=\frac{\sum_{\mathrm{ij}} \frac{\left|I_{n e w, R}(i, j)-\delta_{R, \mathrm{ij}}\right|}{I_{n e w, R}(i, j)+\delta_{R, \mathrm{ij}}}}{M^{*} N} \\
& C M_{G}=\frac{\sum_{\mathrm{ij}} \frac{\left|I_{n e w, G}(i, j)-\delta_{G, \mathrm{ij}}\right|}{I_{\text {new }, G}(i, j)+\delta_{G, \mathrm{ij}}}}{M^{*} N} \\
& C M_{B}=\frac{\sum_{\mathrm{ij}} \frac{\left|I_{\text {new, } B}(i, j)-\delta_{B, \mathrm{ij}}\right|}{I_{n e w, B}(i, j)+\delta_{B, \mathrm{ij}}}}{M^{*} N}
\end{aligned}
$$

5.3: Tính $\mathrm{E}_{\text {avg }}=\mathrm{E}_{\text {avg }}\left\{\mathrm{I}_{\text {new }, \mathrm{R}}, \mathrm{I}_{\text {new,G } \mathrm{G}}, \mathrm{I}_{\text {new }, \mathrm{B}}\right\}$ theo công thức (32). Tính $\mathrm{H}_{\text {avg }}=\mathrm{H}_{\text {avg }}\left\{\mathrm{I}_{\text {new }, \mathrm{R}}, \mathrm{I}_{\text {new, }, \mathrm{G}}, \mathrm{I}_{\text {new }, \mathrm{B}}\right\}$ theo công thức (35)

Trả về: $\mathrm{I}_{\text {new }}$, và các tùy chọn trả về

$$
\mathrm{CM}_{\mathrm{R}}, \mathrm{CM}_{\mathrm{G}}, \mathrm{CM}_{\mathrm{B}}, \mathrm{E}_{\mathrm{avg}}, \mathrm{H}_{\mathrm{avg}}
$$

Bảng 2 là giá trị chỉ số của các ảnh \#1 - \#6, được tính từ các công thức (31) đến (35).

Bảng 2. Giá trị chỉ số ảnh thể hiện.

\begin{tabular}{|c|c|c|c|c|c|}
\hline Ảnh & $\mathbf{C M}_{\mathbf{R}}$ & $\mathbf{C M}_{\mathbf{G}}$ & $\mathbf{C M}_{\mathbf{B}}$ & $\mathbf{E}_{\text {avg }}$ & $\mathbf{H}_{\text {avg }}$ \\
\hline$\# 1$ & 0.1180 & 0.1914 & 0.2482 & 5.9395 & 0.3456 \\
\hline$\# 2$ & 0.0160 & 0.0191 & 0.0314 & 7.315 & 0.8216 \\
\hline$\# 3$ & 0.0154 & 0.0188 & 0.0548 & 7.4847 & 0.8001 \\
\hline$\# 4$ & 0.0256 & 0.0298 & 0.0511 & 7.4536 & 0.8642 \\
\hline$\# 5$ & 0.0170 & 0.0293 & 0.0345 & 7.3092 & 0.8504 \\
\hline$\# 6$ & 0.0273 & 0.0304 & 0.0364 & 3.4443 & 0.2861 \\
\hline
\end{tabular}




\section{IV.4. Các kết quả và luận giải}

Trong phần này chúng tôi trình bày hai kiểm thử, chúng bao gồm:

(A) Phép mờ hóa - biến đổi ảnh sủ dụng phân cụm mò̀ cho tổ hợp kênh ảnh

(B) Thử nghiệm nâng cao độ tương phản sủ dụng biến đổi ảnh trên 2 kênh ảnh (kênh $S$ và kênh $V$ ) trong biểu diễn màu $H S V$.

A. Thực nghiệm để kiểm tra hiệu quả của Phép mờ hóa-biến đổi ảnh sử dụng phân cụm mờ cho tổ hợp kênh ảnh so với phép mờ hóa của [7].

Trong thử nghiệm này, trên từng kênh ảnh riêng rẽ $\mathrm{R}, \mathrm{G}$ và $\mathrm{B}$ chúng tôi thực hiện phép mờ hóa bằng biến đổi S-function được xây dựng trong [6].

Hàm biến đổi $\mathrm{S}(\mathrm{x} ; \mathbf{a}, \mathbf{b}, \mathbf{c})$ với các tham số $\mathbf{a}$ và $\mathbf{c}$ được xác định dựa trên phân tích histogram của kênh ảnh và tham số $\mathbf{b}$ được dò tìm bằng phép cực đại hóa fuzzy entropy.

Thuật toán 1 của chúng tôi thực hiện đồng thời cho 3 kênh ảnh $R, G$ và $B$, tham số $\mathrm{f}_{1}, \mathrm{f}_{2}$ để ước lượng khoảng động mức xám $\left[\mathrm{B}_{1, \mathrm{c}, \mathrm{k}} \mathrm{B}_{2, \mathrm{c}, \mathrm{k}}\right]$ của từng cụm $\mathbf{c}$ ứng với kênh $\mathrm{R}, \mathrm{G}$ và $\mathrm{B}$ được xác định dựa trên thực nghiệm, $\mathrm{f}_{\text {cut }}=0.005$. Thuật toán phân cụm tập vector giá trị điểm ảnh $\left\{\mathrm{I}_{\mathrm{R}}(\mathrm{i}, \mathrm{j}), \mathrm{I}_{\mathrm{G}}(\mathrm{i}, \mathrm{j}), \mathrm{I}_{\mathrm{B}}(\mathrm{i}, \mathrm{j})\right\}$ sử dụng số cụm $\mathrm{C} \in[2,10]$. Trong thực nghiệm này chúng tôi chọn $\mathrm{C}=5$.

Bảng 3. So sánh giá trị $H_{\text {avg }}$ trên kênh $R$, $G$ và $B$ của các ảnh là kết quả của phép mò̀ hóa-biến đổi ảnh

\begin{tabular}{|c|c|c|}
\hline Ảnh & $\begin{array}{c}\mathbf{H}_{\text {avg }} \\
\text { (sử dụng [7]) }\end{array}$ & $\begin{array}{c}\mathbf{H}_{\text {avg }} \\
\text { (sử dụng thuật toán 1) }\end{array}$ \\
\hline$\# 1$ & 0.4478 & 0.4950 \\
\hline$\# 2$ & 0.6931 & 0.7879 \\
\hline$\# 3$ & 0.5736 & 0.7200 \\
\hline$\# 4$ & 0.5822 & 0.7624 \\
\hline$\# 5$ & 0.6227 & 0.8157 \\
\hline$\# 6$ & 0.3374 & 0.3512 \\
\hline
\end{tabular}

Bảng 3 cho chúng ta thấy độ không chắc chắn của phép mờ hóa sử dụng nhiều dải động mức xám của thuật toán đề xuất 1 cao hơn so với phương pháp sử dụng một dải động mức xám của [6]. Điều này cũng phù hợp với trực quan khi quan sát các ảnh mờ hóa minh họa của \#1 và \#2.

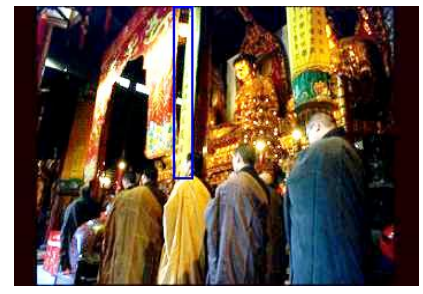

(a)

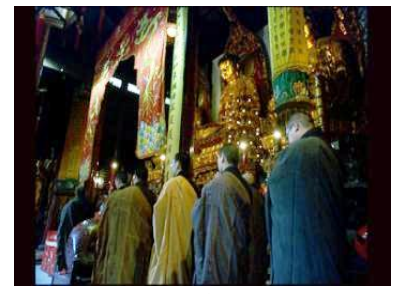

(b)
Hình 9. (a) Anh mò̀ hóa của ảnh \#1 sủ dụng [6]; (b) Ânh kết quả sử dụng thuật toán đề xuất 1

Trên hình ảnh mờ hóa của ảnh \#1 sử dụng biến đổi của [6], chúng ta thấy rõ chi tiết ảnh ở các vùng được đánh dấu hình chữ nhật là bị mất, trong khi đó ảnh biến đổi sử dụng nhiều dải động ước lượng từ phân cụm FCM cho tổ hợp kênh RGB (thuật toán 1) chi tiết ảnh được giữ tốt hơn.

Quan sát các kênh $\mathrm{G}$ và $\mathrm{B}$ của ảnh biến đổi của ảnh \#2 sau đây ta cũng thấy độ chi tiết của ảnh biến đổi sử dụng [6] cũng bị suy giảm nhiều hơn biến đổi ảnh dựa trên thuật toán 1 đã đề xuất.

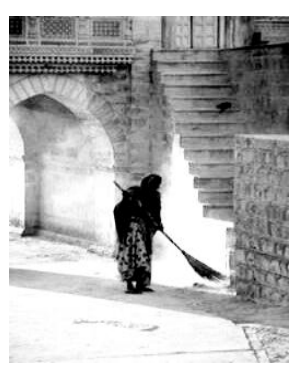

(a)

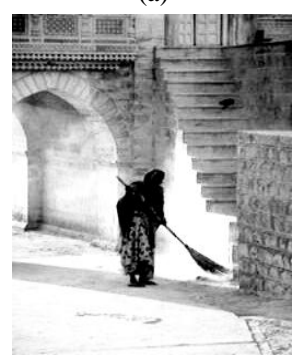

(c)

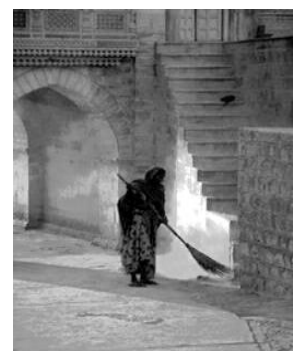

(b)

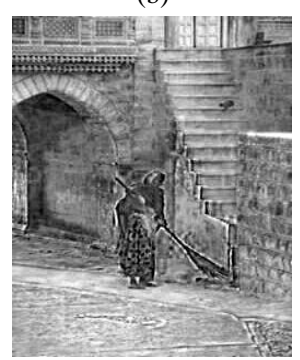

(d)
Hình 10. Kênh $G$ và kênh $B$ của ảnh biến đổi sủ dụng [6] (a), (c). Kênh $G$ và kênh $B$ của ảnh biến đổi sủ dụng thuật toán $1(b),(d)$.

Phân tích trên lại cho thấy việc biến đổi của [6] chỉ sử dụng chỉ một bộ tham số $\left\{\mathbf{a}, \mathbf{b}_{\text {opt }}, \mathbf{c}\right\}$ liên quan đến dải động mức xám của kênh ảnh cho một dạng hàm biến đổi như S-function để biến đổi ảnh có thể làm mất chi tiết ảnh. Trái lại, phương pháp sử dụng nhiều dải động của mức xám $\left\{\mathrm{B}_{1, \mathrm{c}, \mathrm{k}}, \mathrm{B}_{2, \mathrm{c}, \mathrm{k}}\right\}$ được ước lượng 
từ histogram mờ, như của thuật toán 1 đã giữ được chi tiết ảnh tốt hơn.

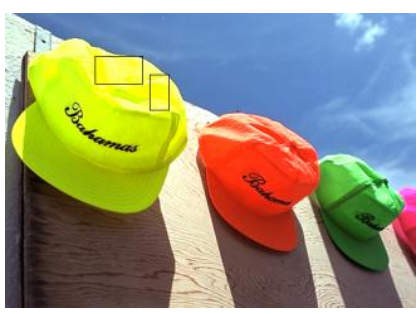

(a)

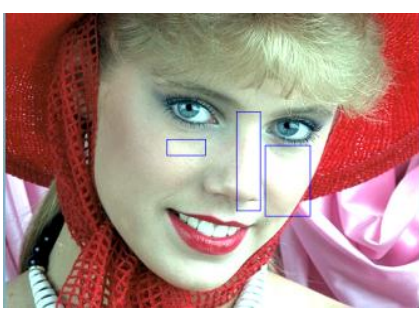

(b)
Hình 11. Sư dụng biến đổi ảnh [6] với một dải động múc xám cho ảnh \#3(a), \#5 (b) và áp dụng [7].

Trên Hình 11, các ảnh kết quả minh họa bị mất chi tiết ảnh, xem ở các vùng chữ nhật đánh dấu. Điều đó chứng tỏ sự hạn chế của việc chỉ dùng một dải động mức xám cho các biến đổi ảnh.

B. Thử nghiệm nâng cao độ tương phản sử dụng thuật toán đề xuất 2 trên hai kênh ảnh $\mathrm{S}$ và $\mathrm{V}$ trong biểu diễn màu HSV của ảnh đầu vào so với sử dụng thủ tục 1 (dựa vào thuật toán gốc).

Trong thử nghiệm này, chúng tôi thực hiện kiểm tra hiệu quả của thuật toán 2 đề xuất sử dụng phép phân cụm FCM để xác định tham số cho phép biến đổi ảnh trong định nghĩa 3.2, công thức (27) so với sử dụng thủ tục 1 trên 2 kênh ảnh $S$ và $V$ riêng rẽ. Các bước được thực hiện như sau:

- Đầu tiên, chuyển biểu diễn màu RGB sang biểu diễn màu HSV của ảnh đầu vào. Phân cụm dữ liệu mức xám tổ hợp kênh $\mathrm{S}$ và kênh $\mathrm{V}$ với tham số được chọn như trong thử nghiệm $\mathbf{A}$ (số cụm $\mathrm{C}=5$ v.v...). trên từng kênh ảnh $\mathrm{S}$ và kênh $\mathrm{V}$ riêng rẽ ước lượng $\left\{\mathrm{B}_{1, \mathrm{c}, \mathrm{k}}, \mathrm{B}_{2, \mathrm{c}, \mathrm{k}}\right\}(\mathrm{k} \in\{\mathrm{S}, \mathrm{V}\})$ và thực hiện biến đổi ảnh cho kênh $\mathrm{S}$ và kênh $\mathrm{V}$ tương ứng sử dụng hàm biến đổi $\mathrm{F}_{\mathrm{S}}, \mathrm{F}_{\mathrm{V}}$ (công thức (27)) có tham số của các hàm biến đổi là các dải động mức xám ước lượng từ thuật toán 1.

- Thứ hai, xác định giá trị độ tương phản theo thuật toán 2 và các bước liên quan cho kênh ảnh $S$ và $\mathrm{V}$ đã biến đổi ở bước thứ nhất với kích thước cửa sổ $3 \times 3$ và tham số $\mathrm{t}=0.25$ (công thức (16)).

- Thứ ba, tổng hợp lại các kênh ảnh $\mathrm{H}$ gốc, kênh $\mathrm{S}$ và kênh $\mathrm{V}$ đã nâng cao độ tương phản, sau đó biến đổi ngược từ biểu diễn HSV sang biểu diễn RGB.
- Cuối cùng, tính giá trị mức xám không thuần nhất tại từng điểm ảnh ứng với các kênh ảnh $R, G$ và $\mathrm{B}$ (các giá trị này được dùng để tính độ đo tương phản trung bình trên từng kênh $\mathrm{R}, \mathrm{G}$ và $\mathrm{B}$ trong bảng 4 ).

Giá trị trung bình độ tương phản trực tiếp $\mathrm{CM}$ được tính trên kênh $\mathrm{R}, \mathrm{G}$ và $\mathrm{B}$ trong biểu diễn màu RGB của ảnh đầu vào được tính với ảnh giá trị mức xám trung bình không thuần nhất của từng kênh $R, G$ và $B$ của ảnh $R G B$ gốc và ảnh kết quả đầu ra khi sử dụng thủ tục 1 và phương pháp đề xuất (thuật toán 2) được thể hiện ở Bảng 4.

Bảng 4. Kết quả chỉ số ảnh đầu ra của thủ tục l(gốc), và thuật toán 2 đề xuất trên tùng kênh ảnh $R, G$ và $B$.

\begin{tabular}{|c|c|c|c|c|c|c|}
\hline \multirow{2}{*}{ Ảnh } & \multicolumn{2}{|c|}{$\mathrm{CM}_{\mathrm{R}}$} & \multicolumn{2}{c|}{$\mathrm{CM}_{\mathrm{G}}$} & \multicolumn{2}{c|}{$\mathrm{CM}_{\mathrm{B}}$} \\
\cline { 2 - 7 } & $\begin{array}{c}\text { Thủ tục } \\
\text { 1 gốc }\end{array}$ & $\begin{array}{c}\text { Thuật } \\
\text { toán 2 }\end{array}$ & $\begin{array}{c}\text { Thủ tục } \\
1 \text { gốc }\end{array}$ & $\begin{array}{c}\text { Thuật } \\
\text { toán 2 }\end{array}$ & $\begin{array}{c}\text { Thủ tục1 } \\
\text { gốc }\end{array}$ & $\begin{array}{c}\text { Thuật } \\
\text { toán 2 }\end{array}$ \\
\hline$\# 1$ & 0.1244 & $\mathbf{0 . 2 1 4 3}$ & 0.1961 & $\mathbf{0 . 2 5 7 5}$ & 0.2511 & $\mathbf{0 . 3 3 7 3}$ \\
\hline$\# 2$ & 0.0157 & $\mathbf{0 . 1 6 7 6}$ & 0.0193 & $\mathbf{0 . 1 6 8 5}$ & 0.0320 & $\mathbf{0 . 1 7 2 8}$ \\
\hline$\# 3$ & 0.0155 & $\mathbf{0 . 2 1 5 7}$ & 0.0192 & $\mathbf{0 . 2 1 6 6}$ & 0.0543 & $\mathbf{0 . 2 3 5 1}$ \\
\hline$\# 4$ & 0.0284 & $\mathbf{0 . 1 2 3 2}$ & 0.0338 & $\mathbf{0 . 1 2 5 4}$ & 0.0570 & $\mathbf{0 . 1 3 8 5}$ \\
\hline$\# 5$ & 0.0170 & $\mathbf{0 . 0 9 6 7}$ & 0.0299 & $\mathbf{0 . 1 0 0 3}$ & 0.0352 & $\mathbf{0 . 1 0 3 3}$ \\
\hline$\# 6$ & 0.0275 & $\mathbf{0 . 0 8 6 1}$ & 0.0307 & $\mathbf{0 . 0 8 7 9}$ & 0.0375 & $\mathbf{0 . 0 8 7 0}$ \\
\hline
\end{tabular}

Bảng 5. Kết quả chỉ số ảnh đầu ra của thủ tục 1(gốc), và thuật toán 2 đề xuất trên tù̀ng kênh ảnh $R, G$ và $B$.

\begin{tabular}{|c|c|c|c|c|}
\hline \multirow{2}{*}{ Ảnh } & \multicolumn{2}{|c|}{$\mathrm{E}_{\mathrm{avg}}$} & \multicolumn{2}{c|}{$\mathrm{H}_{\mathrm{avg}}$} \\
\cline { 2 - 5 } & $\begin{array}{c}\text { Thủ tục } \\
1 \text { gốc }\end{array}$ & $\begin{array}{c}\text { Thuật } \\
\text { toán 2 }\end{array}$ & $\begin{array}{c}\text { Thủ tục } \\
1 \text { gốc }\end{array}$ & $\begin{array}{c}\text { Thuật } \\
\text { toán 2 }\end{array}$ \\
\hline$\# 1$ & 5.8446 & $\mathbf{6 . 1 8 5 5}$ & 0.3456 & $\mathbf{0 . 3 0 7 7}$ \\
\hline$\# 2$ & 6.8158 & $\mathbf{7 . 2 5 6 8}$ & 0.8216 & $\mathbf{0 . 7 0 0 4}$ \\
\hline$\# 3$ & 6.9599 & $\mathbf{7 . 0 0 5 1}$ & 0.8001 & $\mathbf{0 . 6 7 3 7}$ \\
\hline$\# 4$ & 7.2337 & $\mathbf{7 . 4 1 7 9}$ & 0.8642 & $\mathbf{0 . 7 9 4 7}$ \\
\hline$\# 5$ & 7.0103 & $\mathbf{7 . 5 3 7 6}$ & 0.8504 & $\mathbf{0 . 8 2 3 3}$ \\
\hline$\# 6$ & 3.0140 & $\mathbf{4 . 0 1 7 3}$ & 0.2868 & $\mathbf{0 . 2 8 6 1}$ \\
\hline
\end{tabular}

Bảng 4 và 5 của kết quả thực nghiệm của các ảnh \#1 - \#6 đã thể hiện các chỉ số khách quan độ tương phản trực tiếp trên từng kênh $\mathrm{R}, \mathrm{G}$ và $\mathrm{B}$ khi sử dụng thuật toán 2 đều cho kết quả cao hơn khi sử dụng thuật toán [7]. Chỉ số khách quan $\mathrm{E}_{\mathrm{avg}}$ khi áp dụng thuật toán 2 cũng cao hơn so với áp dụng thủ tục 1 . Cũng vậy, chỉ số khách quan $\mathrm{H}_{\text {avg }}$ khi áp dụng thuật toán 2 , ngoài ảnh \#1, đều có giá trị nhỏ hơn so với chỉ số ảnh kết quả khi áp dụng thủ tục 1 . 


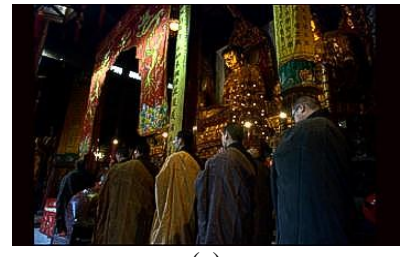

(a)

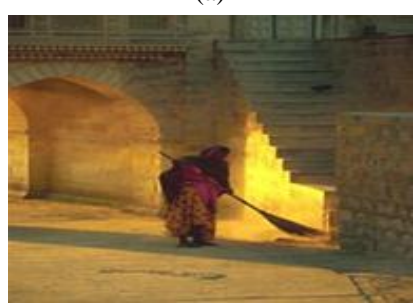

(c)

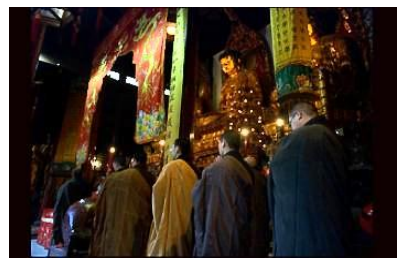

(b)

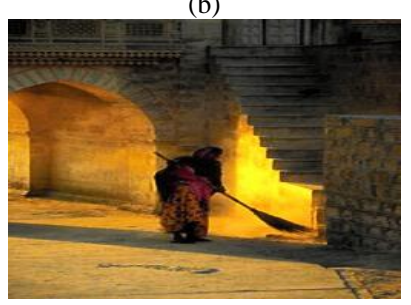

(d)

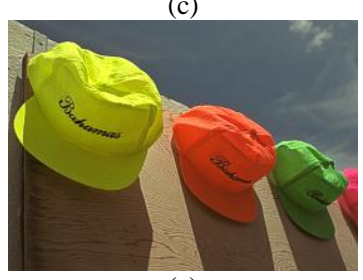

(e)

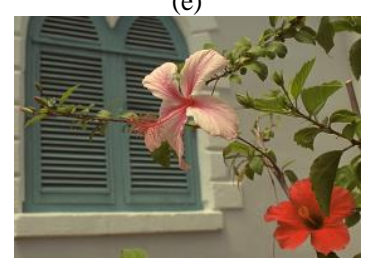

(h)

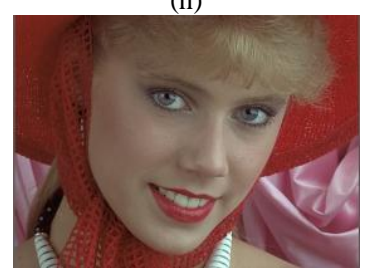

(1)

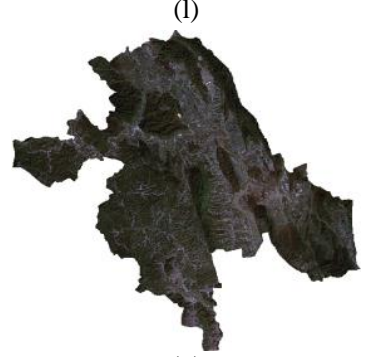

(n)

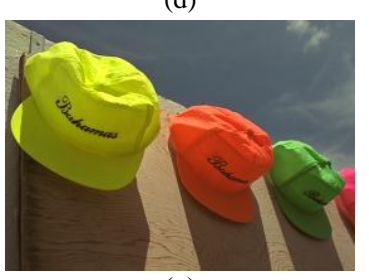

(g)

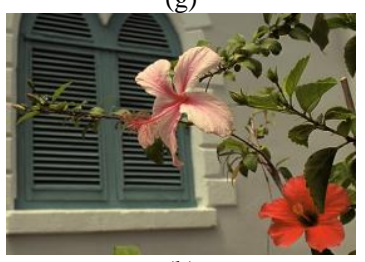

(k)

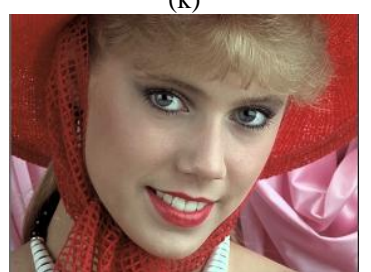

(m)

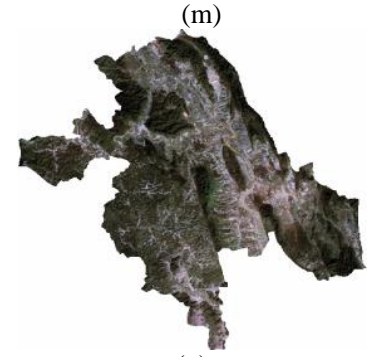

(p)

Hình 12. Anh kết quả (các ảnh cột bên trái) sủ dụng [7] Ảnh kết quả (các ảnh cột bên phải) sủ dụng thuật toán 2.

\section{KẾT LUẬN}

Trong bài báo này chúng tôi đã đề xuất 3 đóng góp mới cho phương pháp nâng cao độ tương phản ảnh màu theo tiếp cận trực tiếp.

Thứ nhất, chúng tôi đề xuất một phương pháp biến đổi ảnh mới sử dụng nhiều hàm $\mathrm{S}$-function được tham số hóa ứng với từng dải động mức xám. Các dải động mức xám này được ước lượng tự động bằng thuật toán phân cụm FCM. Phương pháp này được so sánh trực tiếp với phương pháp biến đổi sử dụng một hàm $\mathrm{S}$ function với tham số hóa dựa trên cực đại fuzzy entropy [6].

Thứ hai, chúng tôi cũng đề xuất một thuật toán mới theo phương pháp nâng cao độ tương phản trực tiếp cho ảnh màu dựa trên thuật toán trong [7].

Cuối cùng, là một cải tiến nhỏ để tăng độ trơn của các giá trị độ thuần nhất của điểm ảnh khi thay đổi cách kết nhập các giá trị địa phương (tại từng điểm ảnh) như gradient, entropy, độ lệch chuẩn trung bình và moment bậc 4 so với cách kết nhập của [7].

Kết qủa thực nghiệm đã chứng tỏ rằng các kỹ thuật đề xuất của chúng tôi làm việc tốt với đa dạng ảnh màu. Ảnh nâng cao độ tương phản trơn và sự thay đổi mức độ sáng trong khi vẫn bảo toàn chi tiết ảnh gốc tốt hơn so với phương pháp được công bố trong $[6,7]$. Độ sáng của các kênh ảnh sau khi nâng cao độ tương phản tương đồng với độ sáng ảnh đầu ra của các phương pháp gián tiếp. Trong các nghiên cứu tiếp theo, chúng tôi sẽ tiếp tục nghiên cứu các dạng kết nhập khác của các đặc trưng địa phương như gradient, entropy, độ lệch trung bình và moment bậc 4 dạng $\mathrm{HO}_{\mathrm{ij}}=\mathrm{f}\left(\mathrm{E}_{\mathrm{ij}}, \mathrm{H}_{\mathrm{ij}}, \mathrm{V}_{\mathrm{ij}}, \mathrm{R}_{4, \mathrm{ij}}\right)$ khi tính độ thuần nhất tại từng điểm ảnh.

\section{TÀI LIỆU THAM KHẢO}

[1] S. S. AGAiAN, S. BLAIR and K. A. PANETTA, "Transform coefficient histogram-based image enhancement algorithms using contrast entropy", IEEE Trans. Image Processing, vol. 16, no. 3, (2007): 741-758.

[2] ARICI T., DIKBAS S., and ALTUNBASAK Y., " $A$ Histogram Modification Framework and Its Application for Image Contrast Enhancement," IEEE Transactions on Image Processing, vol. 18, no. 9, (2009):1921-1935. 
[3] A. BEGHDADI, A.L. NEGRATE, "Contrast enhancement technique based on local detection of edges", Comput. Vision Graphics Image Process. 46 (1989):162-174.

[4] BEZDEK, JAMES C. Pattern recognition with fuzzy objective function algorithms. Springer Science \& Business Media, (2013).

[5] A.O. BOUDRAA and E. H. S. DIOP, "Image contrast enhancement based on 2D teager-kaiser operator", Proc. of the IEEE International Conference on Image Processing, (2008.): 3180-3183.

[6] CHENG H.D, HUIJUAN XU, "A novel fuzzy logic approach to contrast enhancement", Pattern Recognition 33 (2000):809-819.

[7] CHENG H.D., MEI XUE, SHI X,J., “Contrast enhancement based on a novel homogeneity measurement", Pattern Recognition 36 (2003):2687 2697.

[8] L.DASH, B.N. CHATTERJI, "Adaptive contrast enhancement and de-enhancement", Pattern Recognition 24 (1991) :289-302.

[9] A.P. DHNAWAN, G. BUELLONI, R. GORDON, Enhancement of mammographic features by optimal adaptive neighborhood image processing, IEEE Trans. Med. Imaging 5 (1986):8-15.

[10] M. M. GUPTA, J. QI, “Theory of T-norms and fuzzy inference methods", Fuzzy Sets and Systems 40, (1991):431-450.

[11] HANMANDLU M., DEVENDRA JHA, ROCHAK, "Color image enhancement by fuzzy intensification", Pattern Recognition Letters 24 (2003):81-87.

[12] HANMANDLU M, DEVENDRA JHA, “An Optimal Fuzzy System for Color Image Enhancement”, IEEE
Transactiong on Image Processiong, Vol. 15, No.10 (2006): 2956-2966.

[13] S. LEE, "An efficient content-based image enhancement in the compressed domain using Retinex theory", IEEE Trans. Circuits and Systems for Video Technology, vol. 17, no. 2, pp. (2007):199-213.

[14] PONOMARENKO N. PONOMARENKO, L. JIN, O. IEREMEIEV, V. LUKIN, K. EGIAZARIAN, J. ASTOLA, B. VOZEL, K. CHEHDI, M. CARLI, F.BATTISTI, C.-C. JAY KUO, "Image database TID2013: Peculiarities, results and perspectives, Signal Processing", Imag Communication, vol. 30, Jan. (2015):57-77.

[15] M. SHAKERI, M.H. DEZFOULIAN, H. KHOTANLOU, A.H. BARATI, Y. MASOUMI, Image contrast enhancement using fuzzy clustering with adaptive cluster parameter and sub-histogram equalization, Digital Signal Processing 62, (2017) :224-237.

[16] SHEN-CHUAN TAI, TING-CHOU TSAI, YI-YING CHANG, WEI-TING TSAI AND KUANG-HUI TANG, "Contrast Enhancement through Clustered Histogram Equalization", Research Journal of Applied Sciences, Engineering and Technology 4(20), (2012):3965-3968, ISSN: 2040-7467.

[17] M. J. SOHA AND A. A. SCHWARTZ, "Multidimensional histogram normalization contrast enhancement," in Proc. 5th Canad. Symp.. Remote Sensing, (1978):86-93.

Nhận bài ngày: 12/01/2017 


\section{SƠ LƯợC VỀ TÁC GIẢ}

\section{NGUYẼ̃N VĂN QUYỀN}

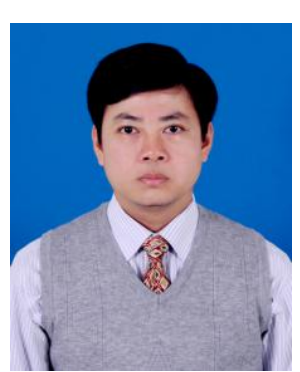

Sinh năm 1979 tại Hải Phòng.

Nhận bằng thạc sĩ chuyên ngành Khoa học máy tính tại trường $Đ H$ Sư phạm Hà Nội, năm 2008 Đang là NCS tại Viện CNTT Viện Hàn lâm KH\&CN Việt Nam Hiện công tác tại Phòng QLSĐH, trường ĐH Hải Phòng

Lĩnh vực nghiên cứu: Xử lý ảnh, khai phá dữ liệu, tính toán mềm

Điện thoại: 0904.815.444

Email: quyenqlkh.dhhp@gmail.com

\section{TRÀ̀ THÁI SƠN}

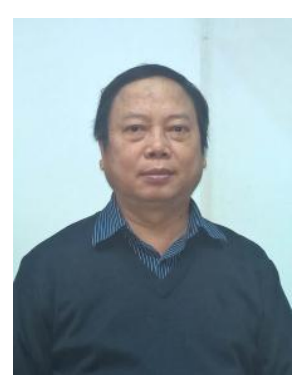

Sinh năm 1955, tại Nghệ An

Nhận học vị tiến sĩ năm 1990, tại

Viện hàn lâm Khoa học Liên Xô

Hiện công tác tại Viện CNTT, Viện Hàn lâm KH\&CN Việt Nam

Lĩnh vực nghiên cứu: Lập luận xấp xỉ, tính toán mềm

Điện thoại: 0903409894

Mail: ttson@ioit.ac.vn

\section{NGUYẼ̃N TÂN ÂN}

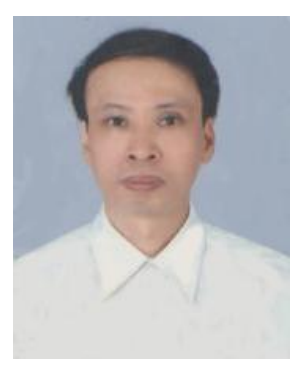

Sinh năm 1953 tại Hà Nội.

Nhận bằng tiến sĩ năm 2002, tại trường ĐH Năng lượng Maxcơva; nhận học hàm PGS năm 2012 tại Viêt Nam.

Hiện công tác tại Khoa CNTT, Học viện Quản lý Giáo dục. Lĩnh vực nghiên: Trí tuệ nhân tạo, Mạng nơron nhân tạo, Tính toán mềm, Giáo dục tin học

Điện thoại: 0912321559

Email: nguyentanan@yahoo.com

\section{NGÔ HOÀNG HUY}

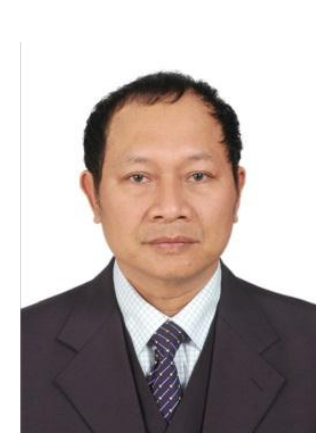

Sinh năm 1969 tại Hà Nội.

Tốt nghiệp chuyên ngành Toán tin tại trường ĐH Sư phạm Hà Nội năm 1990.

Hiện công tác tại Viện CNTT, Viện Hàn lâm KH\&CN Việt Nam

Lĩnh vực nghiên cứu: Xử lý ảnh, Xử lý tiếng nói, Trí tuệ nhân tạo, Khai phá dữ liệu, Hệ thống nhúng.

Điện thoại: 0904140022

Email: huyngo3i@gmail.com

\section{ĐẶNG DUY AN}

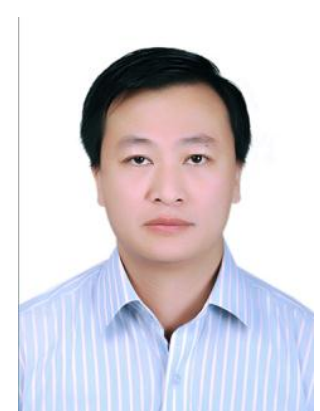

Sinh năm 1974 tại Thái Nguyên. Nhận bằng thạc sĩ tại Đại học Thái Nguyên, năm 2014.

Hiện công tác tại Công ty tập đoàn CDC CORP - Viện máy và Dụng cụ Công nghiệp - Bộ Công Thương

Lĩnh vực nghiên cứu: Trí tuệ nhân tạo, khai phá dữ liệu, hệ thống nhúng

Điện thoại: 0913215111

Mail: duyanimi@gmail.com 